\title{
A REWRITING VIEW OF SIMPLE TYPING
}

\author{
AARON STUMP ${ }^{a}$, HANS ZANTEMA $^{b}$, GARRIN KIMMELL $^{c}$, AND RUBA EL HAJ OMAR \\ ${ }^{a, c, d}$ Computer Science, The University of Iowa \\ e-mail address: astump@acm.org, \{garrin-kimmell,roba-elhajomar\}@uiowa.edu \\ ${ }^{b}$ Department of Computer Science, TU Eindhoven, The Netherlands; and Institute for Computing \\ and Information Sciences, Radboud University, The Netherlands \\ e-mail address: h.zantema@tue.nl
}

\begin{abstract}
This paper shows how a recently developed view of typing as small-step abstract reduction, due to Kuan, MacQueen, and Findler, can be used to recast the development of simple type theory from a rewriting perspective. We show how standard meta-theoretic results can be proved in a completely new way, using the rewriting view of simple typing. These meta-theoretic results include standard type preservation and progress properties for simply typed lambda calculus, as well as generalized versions where typing is taken to include both abstract and concrete reduction. We show how automated analysis tools developed in the term-rewriting community can be used to help automate the proofs for this meta-theory. Finally, we show how to adapt a standard proof of normalization of simply typed lambda calculus, for the rewriting approach to typing.
\end{abstract}

\section{INTRODUCTION}

This paper develops a significant part of the theory of simple types based on a recently introduced rewriting approach to typing. The idea of viewing typing as a small-step abstract reduction relation was proposed by Kuan, MacQueen, and Findler in 2007, and explored also by Ellison, Şerbănuţă, and Roşu [13, 9, 14]. These works sought to use rewrite systems to specify typing in a finer-grained way than usual type systems. Our motivation is more foundational: we seek to prove standard meta-theoretic properties of type systems directly, based on the rewriting formulation. The goal is to develop new methods which could provide a different perspective on familiar type systems, and perhaps yield new results for more advanced type systems.

Our focus in this paper is simple type systems, where the central typing construct is the function type $T \Rightarrow T^{\prime}$. We will view such types as abstractions of functions, and incrementally rewrite (typable) functions to such function types, using an abstract small-step

2012 ACM CCS: [Software and its Engineering]: Software notations and tools - Formal language definitions - Syntax.

Key words and phrases: Term rewriting, Type safety, Confluence.

This work was partially supported by the U.S. National Science Foundation, contract CCF-0910510, as part of the Trellys project.

DOI:10.2168/LMCS-9(1:04)2013
(C) A. Stump, H. Zantema, G. Kimmell, and R. El Haj Omar (C) Creative Commons 
reduction relation. It will be straightforward to prove the standard property of type safety, based on type preservation and progress, using this rewriting formulation. This viewpoint also allows us to combine the usual concrete reduction relation and our new abstract reduction relation together, simply by taking their set-theoretic union. We will prove that this combined reduction relation is confluent for typable terms, defined as terms which reduce, using abstract steps, to a type. To prove both type preservation and confluence we use observations developed in the context of abstract reduction systems. We then develop our final main result, which is a proof of normalization for the simply typed lambda calculus, based on the rewriting approach. This proof has several novel features, which shed new light on the reducibility semantics of types used in standard proofs of normalization.

This paper expands in several important ways on a previous paper of Stump, Kimmell, and El Haj Omar, which was presented at RTA 2011 [20]:

- We use the rewriting method to prove type preservation for full $\beta$-reduction; the RTA '11 paper showed it only for call-by-value computation.

- We prove preservation for a new notion we call generalized typing, where concrete and abstract reduction steps can be intermixed. This generalizes the so-called direct computation rules of the well-known NuPRL system [2].

- We correct an error in the RTA '11 paper, where we claimed that type preservation is a corollary of confluence for typable terms. In fact, confluence is a straightforward corollary of type preservation.

- We have shown how a standard proof of normalization for simply typable terms is adapted to the rewriting approach to typing. This adaptation reveals an interesting perspective on types as abstractions of terms.

- Due to the amount of new material, we have dropped the treatment of several variants of STLC, which are studied in the RTA paper.

As Zantema had a substantial contribution to these extensions, he was added as an author.

The remainder of the article is organized as follows. Section 2 provides a brief introduction to abstract reduction systems as used later in the paper. Section 3 gives a standard presentation of the simply typed lambda calculus along with the fundamental meta-theoretic properties. Section 4 recasts the simply typed lambda calculus static and operational semantics within the framework of abstract reduction systems. Section 5 gives some abstract reduction theory to be used in Section 6 where type preservation and confluence is proved. Section 7 then proves progress and type safety. Section 8 proves type preservation and confluence for a system with uniform syntax for types and term. For this result, we use automated tools developed in the term-rewriting community, to verify some of the properties necessary for applying theorems proved in Section 5. Section 9 extends these to a generalized notion of typing, based on the union of the concrete and abstract reduction relations. Section 10 applies a rewriting approach to prove the normalization of well-typed simply typed lambda calculus terms. We conclude and identify future directions in Section 11.

\section{Rewriting Preliminaries}

In this section we collect some basic properties in the setting of abstract reduction systems. That is, we consider relations $\rightarrow$ being a subset of $X \times X$ for some arbitrary set $X$.

We write $\cdot$ for relation composition, and inductively define $\rightarrow^{0}=i d$ (the identity) and $\rightarrow^{n}=\rightarrow^{n-1} \cdot \rightarrow$ for $n>0$. As usual, for a relation $\rightarrow$ we write $\leftarrow$ for its reverse, $\rightarrow=$ for its 
reflexive closure (zero or one times), $\rightarrow^{+}=\bigcup_{i=1}^{\infty} \rightarrow^{i}$ for its transitive closure (one or more times), and $\rightarrow^{*}=\bigcup_{i=0}^{\infty} \rightarrow^{i}$ for its transitive reflexive closure (zero or more times). We will also use standard notation $R(A)$ for the image of set $A$ under relation $R$ :

$$
R(A)=\left\{a^{\prime} \mid \exists a \in A .\left(a, a^{\prime}\right) \in R\right\}
$$

We can use this notation to denote the set of predecessors of a set $A$ with respect to $\rightarrow$ as $\leftarrow^{*}(A)$. We will also write $I d_{A}$ for $\{(a, a) \mid a \in A\}$.

A relation $\rightarrow$ is said to

- be confluent (Church Rosser, $C R(\rightarrow)$ ) if $\leftarrow^{*} \cdot \rightarrow^{*} \subseteq \rightarrow^{*} \cdot \leftarrow^{*}$,

- be locally confluent (Weak Church Rosser, $W C R(\rightarrow))$ if $\leftarrow \cdot \rightarrow \subseteq \rightarrow^{*} \cdot \leftarrow^{*}$,

- have the diamond property $(\diamond(\rightarrow))$ if $\leftarrow \cdot \rightarrow \subseteq \rightarrow^{=} \cdot \leftarrow^{=}$,

- be deterministic $(\operatorname{det}(\rightarrow))$ if $\leftarrow \cdot \rightarrow \subseteq i d$.

- be terminating if there is no infinite descending chain $a_{1} \rightarrow a_{2} \rightarrow \cdots$.

- be convergent if it is confluent and terminating.

We will sometimes also call an element $x_{1} \in X$ confluent iff for all $x_{2}, x_{3} \in X$ with $x_{1} \rightarrow^{*} x_{2}$ and $x_{1} \rightarrow^{*} x_{3}$, there exists $x_{4} \in X$ with $x_{2} \rightarrow^{*} x_{4}$ and $x_{3} \rightarrow^{*} x_{4}$. It is well-known and easy to see that $\operatorname{det}(\rightarrow) \Rightarrow \diamond(\rightarrow) \Rightarrow C R(\rightarrow) \Rightarrow W C R(\rightarrow)$.

Finally, if $\rightarrow_{a}$ and $\rightarrow_{b}$ are binary relations, below we will often write $\rightarrow_{b a}$ for $\rightarrow_{a} \cup \rightarrow_{b}$.

\section{A Standard Presentation of Simple Typing}

In this section, we summarize a standard presentation of the simply typed lambda calculus (STLC), including syntax and semantics, and statements of the basic meta-theoretic properties of type preservation and progress. Sections 4 and following will recapitulate this development in detail, from the rewriting perspective. Including some type and term constants, together with reduction rules for them, is very standard in the study of programming languages and typed lambda calculus. One example is Mitchell's treatment of STLC with additional rules [16, Section 4.4.3]). For progress, it is indeed instructive to include reduction rules for some selected constants. Otherwise, there are no stuck terms that should be ruled out by the type system, since in pure STLC, every closed normal form is a value, namely a $\lambda$-abstraction. We treat additional rules representatively (as opposed to parametrically), using constants $a$ and $f$ below.

3.1. Syntax and Semantics. The syntax for terms, types, and typing contexts is the following, where $A, f$, and $a$ are specific constants, and $x$ ranges over a countably infinite set of variables:

$$
\begin{array}{ll}
\text { types } T & ::=A \mid T_{1} \Rightarrow T_{2} \\
\text { standard terms } t & ::=f|a| x\left|t_{1} t_{2}\right| \lambda x: \text { T.t } \\
\text { typing contexts } \Gamma & ::=\cdot \mid \Gamma, x: T
\end{array}
$$

We will write Types for the set of all types. We assume standard additional conventions and notations, such as $[t / x] t^{\prime}$ for the capture-avoiding substitution of $t$ for $x$ in $t^{\prime}$, and $E[t]$ for grafting a term into an evaluation context. Figure 1 defines a standard type system for STLC. The judgments derived by the rules in the figure are of the form $\Gamma \vdash t: T$, which can be viewed as deterministically computing a type $T$ as output, given a term $t$ and a typing context $\Gamma$ as inputs. In the topmost leftmost rule of the Figure, we use the notation 


$$
\begin{array}{lll}
\frac{\Gamma(x)=T}{\Gamma \vdash x: T} & \overline{\Gamma \vdash f: A \Rightarrow A} & \overline{\Gamma \vdash a: A} \\
\frac{\Gamma \vdash t_{1}: T_{2} \Rightarrow T_{1} \quad \Gamma \vdash t_{2}: T_{2}}{\Gamma \vdash t_{1} t_{2}: T_{1}} & \frac{\Gamma, x: T_{1} \vdash t: T_{2}}{\Gamma \vdash \lambda x: T_{1} \cdot t: T_{1} \Rightarrow T_{2}}
\end{array}
$$

Figure 1: Type-computation rules for STLC with selected constants

$$
\begin{aligned}
& \overline{E\left[(\lambda x: T . t) t^{\prime}\right] \rightarrow E\left[\left[t^{\prime} / x\right] t\right]} \quad \text { values } v::=\lambda x: \text { T.t }|a| f \\
& \overline{E[f a] \rightarrow E[a]} \\
& \text { evaluation contexts } E::=*|(E t)|(t E) \mid \lambda x: T . E
\end{aligned}
$$

Figure 2: Small-step reduction semantics for STLC

$\Gamma(x)=T$ to mean that there is a binding $x: T$ in $\Gamma$. We assume there is at most one such binding in $\Gamma$, renaming bound variables as necessary to ensure this. A standard small-step reduction semantics, for unrestricted $\beta$-reduction, is defined using the rules of Figure 2 . Following standard usage, terms of the form $\left(\lambda x:\right.$ T.t) $t^{\prime}$ or $f a$ are called redexes. An example of a concrete reduction is (with redexes underlined):

$$
\underline{(\lambda x:(A \rightarrow A) \cdot x(x a)) f} \rightarrow f \underline{(f a)} \rightarrow \underline{f a} \rightarrow a
$$

3.2. Basic Meta-theory. The main theorem relating the reduction relation $\rightarrow$ and typing is type preservation, which states the following, either for unrestricted $\beta$-reduction $\rightarrow$ or for some restriction of $\rightarrow$ (as we will consider below):

$$
\left(\Gamma \vdash t: T \wedge t \rightarrow t^{\prime}\right) \Rightarrow \Gamma \vdash t^{\prime}: T
$$

The standard proof method is to proceed by induction on the structure of the typing derivation, with case analysis on the reduction derivation (cf. Chapters 8 and 9 of [17]). A separate induction is required to prove a substitution lemma, needed critically for type preservation for $\beta$-reduction steps:

$$
\Gamma \vdash t: T \wedge \Gamma, x: T \vdash t^{\prime}: T^{\prime} \Rightarrow \Gamma \vdash[t / x] t^{\prime}: T^{\prime}
$$

For call-by-value programming languages, one also typically proves progress, formulated in terms of values:

$$
(\cdot \vdash t: T \wedge t \nrightarrow \rightarrow) \Rightarrow t \in \text { values }
$$

Here, the notation $t \nrightarrow \rightarrow$ means $\forall t^{\prime} . \neg\left(t \rightarrow t^{\prime}\right)$; i.e., $t$ is a normal form. Normal forms which are not values are called stuck terms. An example is $f f$. Combining type preservation and progress allows us to prove type safety [24]. This property states that the normal forms of closed well-typed terms are values, not stuck terms, and in our setting can be stated:

$$
\left(\cdot \vdash t: T \wedge t \rightarrow^{*} t^{\prime} \not \rightarrow\right) \Rightarrow \exists v \cdot t^{\prime}=v
$$

This is proved by induction on the length of the reduction sequence from $t$ to $t^{\prime}$. As already noted, without constants ( $f$ and $a$ here), this result is not so interesting for STLC, since it follows already by simpler reasoning: reduction cannot introduce new free variables, so $t^{\prime}$ 


$\begin{array}{lll}\text { types } T & ::= & A \mid T_{1} \Rightarrow T_{2} \\ \text { standard terms } t & ::= & x|\lambda x: T . t| t t^{\prime}|a| f \\ \text { mixed terms } m & ::= & x|\lambda x: T . m| m m^{\prime}|a| f \mid \\ & & A \mid T \Rightarrow m \\ \text { standard values } v & ::= & \lambda x: \text { T.t }|a| f \\ \text { mixed values } u & ::= & \lambda x: \text { T.m }|T \Rightarrow m| A|a| f\end{array}$

Figure 3: Syntax for STLC using mixed terms

$$
\begin{aligned}
& \overline{E_{c}\left[\begin{array}{llll}
f & a
\end{array} \rightarrow_{c} \quad E_{c}[a]\right.} c(f-\beta) \\
& \overline{E_{c}[(\lambda x: T . m) u] \rightarrow_{c} E_{c}[[u / x] m]} c(\beta)
\end{aligned}
$$

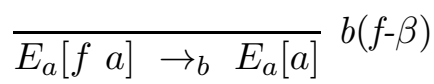

$$
\begin{aligned}
& \overline{E_{a}\left[(\lambda x: T . m) m^{\prime}\right] \rightarrow_{b} E_{a}\left[\left[m^{\prime} / x\right] m\right]} b(\beta) \\
& \overline{E_{a}[(T \Rightarrow m) T] \rightarrow_{a} E_{a}[m]} a(\beta) \\
& \overline{E_{a}[\lambda x: T . m] \rightarrow_{a} E_{a}[T \Rightarrow[T / x] m]} a(\lambda) \\
& \overline{E_{a}[f] \rightarrow_{a} E_{a}[A \Rightarrow A]} a(f) \\
& \overline{E_{a}[a] \rightarrow_{a} E_{a}[A]} a(a) \\
& \text { call-by-value evaluation contexts } E_{c}::=*\left|\left(E_{c} m\right)\right|\left(u E_{c}\right) \\
& \text { unrestricted evaluation contexts } E_{a}::=*\left|\left(E_{a} m\right)\right|\left(m E_{a}\right)\left|\lambda x: T . E_{a}\right| T \Rightarrow E_{a}
\end{aligned}
$$

Figure 4: Concrete call-by-value reduction $\left(\rightarrow_{c}\right)$, concrete full $\beta$-reduction $\left(\rightarrow_{b}\right)$, and abstract reduction $\left(\rightarrow_{a}\right)$ for STLC

must be closed; and it is then easy to prove that closed normal forms are $\lambda$-abstractions, and hence values by definition.

\section{Simple Typing as Abstract Reduction}

In this section, we see how to view a type-computation (also called type-synthesis) system for STLC as an abstract operational semantics. We view function types $T_{1} \Rightarrow T_{2}$ as abstract functions from $T_{1}$ to $T_{2}$, and allow these to be applied to arguments. When $T_{1} \Rightarrow T_{2}$ is applied to the abstract term $T_{1}$, an abstract $\beta$-reduction step is possible, simulating concrete $\beta$-reduction for any function of type $T_{1} \Rightarrow T_{2}$ applied to an argument of type $T_{1}$. Thus, we will see abstract reduction as truly an abstraction of the usual reduction, which we thus view, in contrast, as concrete.

To view typing as an abstract form of reduction, we use mixed terms, defined in Figure 3. Types like $T_{1} \Rightarrow T_{2}$ will serve as abstractions of $\lambda$-abstractions. For our development below, we are going to consider both unrestricted $\beta$-reduction, and also call-by-value $\beta$-reduction, a common restriction implemented in practical functional programming languages like OCAML. Figure 4 gives rules for concrete call-by-value reduction $\left(\rightarrow_{c}\right)$, concrete

full $\beta$-reduction $\left(\rightarrow_{b}\right)$, and abstract reduction $\left(\rightarrow_{a}\right)$. As above, we will refer to any term of the form displayed in context on the left hand side of the conclusion of a rule as a redex. We 
denote the union of these reduction relations as $\rightarrow_{c a}$. The definition of call-by-value evaluation contexts $E_{c}$ enforces left-to-right evaluation order in a standard way, while unrestricted evaluation contexts $E_{a}$ make abstract reduction and full $\beta$-reduction non-deterministic: reduction is allowed anywhere inside a term. This is different from the approach followed by Kuan et al., where abstract and concrete reduction are both deterministic. Here is an example of reduction using the abstract operational semantics:

$$
\begin{aligned}
& \lambda x:(A \Rightarrow A) \cdot \lambda y: A \cdot(x(x y)) \rightarrow_{a} \\
& \lambda x:(A \Rightarrow A) \cdot A \Rightarrow(x(x A)) \rightarrow_{a} \\
& (A \Rightarrow A) \Rightarrow A \Rightarrow((A \Rightarrow A)((A \Rightarrow A) A)) \rightarrow_{a} \\
& (A \Rightarrow A) \Rightarrow A \Rightarrow((A \Rightarrow A) A) \rightarrow_{a} \\
& (A \Rightarrow A) \Rightarrow A \Rightarrow A \rightarrow_{a}
\end{aligned}
$$

The final result is a type $T$, which does not reduce (as noted below). Indeed, using the standard typing rules of Section 3.1 we can prove that the starting term of this reduction has that type $T$, in the empty typing context. Abstract reduction to a type plays the role of typing above.

Lemma 4.1. For all types $T$, we have $T \nrightarrow_{a}$.

Proof. This follows by induction on $T$ and inspection of the rules for $\rightarrow_{a}$.

If we look back at our standard typing rules (Figure 1), we can now see them as essentially big-step abstract operational rules. Recall that big-step call-by-value operational semantics for STLC includes this rule (as well as several others which we elide):

$$
\frac{t_{1} \Downarrow \lambda x: T . t_{1}^{\prime} \quad t_{2} \Downarrow t_{2}^{\prime} \quad\left[t_{2}^{\prime} / x\right] t_{1}^{\prime} \Downarrow t^{\prime}}{t_{1} t_{2} \Downarrow t^{\prime}}
$$

In our setting, big-step call-by-value semantics would be seen as a concrete big-step reduction, which we might denote $\Downarrow_{c}$. The abstract version of this rule, where we abstract $\lambda$-abstractions by arrow-types, is

$$
\frac{t_{1} \Downarrow_{a} T \Rightarrow T^{\prime} \quad t_{2} \Downarrow_{a} T}{t_{1} t_{2} \Downarrow_{a} T^{\prime}}
$$

If we drop the typing context from the standard typing rule for applications (in Figure 1), we obtain essentially the same rule.

The standard approach to proving type preservation relates a small-step concrete operational semantics with a big-step abstract operational semantics (i.e., the standard typing relation). We find it both more elegant, and arguably more informative to relate abstract and concrete small-step relations, as we will do in Section 6 below.

4.1. Rewriting Properties of Abstract Reduction. In this subsection, we study the properties of abstract reduction from the perspective of the theory of abstract reduction systems (ARSs). From this point of view, abstract reduction is very well behaved: it is a convergent ARS, as the following two theorems show.

Theorem 4.2 (Termination of Abstract Reduction). The relation $\rightarrow_{a}$ is terminating. 
Proof. We recursively define a natural-number measure $\mu(m)$ which can be confirmed to reduce from $m$ to $m^{\prime}$ whenever $m \rightarrow_{a} m^{\prime}$ :

$$
\begin{aligned}
\mu(x) & =1 \\
\mu(\lambda x: T . m) & =1+\mu(m) \\
\mu\left(m m^{\prime}\right) & =1+\mu(m)+\mu\left(m^{\prime}\right) \\
\mu(a) & =1 \\
\mu(f) & =1 \\
\mu(A) & =0 \\
\mu(T \Rightarrow m) & =\mu(m)
\end{aligned}
$$

Theorem 4.3. The relation $\rightarrow_{a}$ is confluent.

Proof. In fact, we will prove $\rightarrow_{a}$ has the diamond property (and hence is confluent). Suppose $m \rightarrow_{a} m_{1}$ and $m \rightarrow_{a} m_{2}$. No critical overlap is possible between these steps, because none of the redexes in the $a$-rules of Figure 4 (such as $(T \Rightarrow m) T$ in the $a(\beta)$ rule) can critically overlap another such redex. If the positions of the redexes in the terms are parallel, then (as usual) we can join $m_{1}$ and $m_{2}$ by applying to each the reduction required to obtain the other. Finally, we must consider the case of non-critical overlap (where the position of one redex in $m$ is a prefix of the other position). We can also join $m_{1}$ and $m_{2}$ in this case by applying the reduction to $m_{i}$ which was used in $m \rightarrow_{a} m_{3-i}$, because abstract reduction cannot duplicate or delete an $a$-redex. The only duplication of any subterm in the abstract reduction rules of Figure 4 is of the type $T$ in $a(\lambda)$. The only deletion possible is of the type $T$ in $a(\beta)$. Since types cannot contain redexes, there is no duplication or deletion of redexes. This means that if the position of the first redex is a prefix of the second (say), then there is exactly one descendant (see Section 4.2 of [22]) of the second redex in $m_{1}$, and this can be reduced in one step to join $m_{1}$ with the reduct of $m_{2}$ obtained by reducing the first redex. So every aa-peak can be completed with one joining step on each side of the diagram. This gives the diamond property (and thus confluence for $\rightarrow_{a}$ ).

4.2. Relation with Standard Typing. In this subsection, we prove the following theorem, which relates our notion of typing with the standard one. The proof begins after the statement of some simple auxiliary lemmas, whose proofs are routine and omitted. The proof of the right-to-left direction of the implication will take advantage of the fact that abstract reduction is convergent, as proved in the previous subsection.

Theorem 4.4. For standard terms $t$, a typing judgment $x_{1}: T_{1}, \cdots, x_{n}: T_{n} \vdash t: T$ holds iff $\left[T_{1} / x_{1}, \cdots, T_{n} / x_{n}\right] t \rightarrow{ }_{a}^{*} T$.

Lemma 4.5. If $t_{1} \rightarrow_{a}^{k} T$, then $t_{1} t_{2} \rightarrow_{a}^{k} T t_{2}$.

Lemma 4.6. If $t_{2} \rightarrow_{a}^{k} T$, then $t_{1} t_{2} \rightarrow_{a}^{k} t_{1} T$.

Lemma 4.7. If $t \rightarrow{ }_{a}^{k} T^{\prime}$, then $T \Rightarrow t \rightarrow_{a}^{k} T \Rightarrow T^{\prime}$. 
Proof of Theorem 4.4, left-to-right. Suppose $x_{1}: T_{1}, \cdots, x_{n}: T_{n} \vdash t: T$. We will now prove $\left[T_{1} / x_{1}, \cdots, T_{n} / x_{n}\right] t \rightarrow{ }_{a}^{*} T$ by induction on the structure of the typing derivation of $t$. To simplify the writing of the proof, we will use the following notation:

$$
\begin{aligned}
& \Gamma=x_{1}: T_{1}, \cdots, x_{n}: T_{n} \\
& \Gamma_{\text {sub }}=\left[T_{1} / x_{1}, \cdots, T_{n} / x_{n}\right]
\end{aligned}
$$

\section{Base Case:}

$$
\frac{\Gamma(x)=T}{\Gamma \vdash x: T}
$$

There must be some $i \in\{1, \ldots, n\}$ such that $x=x_{i}$ and $T=T_{i}$. So $\Gamma_{\text {sub }} x=T_{i} \rightarrow_{a}^{*} T_{i}$ as required.

\section{Base Case:}

$$
\overline{\Gamma \vdash f: A \Rightarrow A}
$$

We indeed have $f \rightarrow_{a}(A \Rightarrow A)$, as required. The case for $a: A$ is similar.

Case:

$$
\frac{\Gamma \vdash t_{1}: T_{2} \Rightarrow T_{1} \quad \Gamma \vdash t_{2}: T_{2}}{\Gamma \vdash t_{1} t_{2}: T_{1}}
$$

By the induction hypotheses for the derivations given for the two premises of this rule, we have:

$$
\begin{aligned}
& \Gamma_{\text {sub }} t_{1} \rightarrow_{a}^{*} T_{2} \Rightarrow T_{1} \\
& \Gamma_{\text {sub }} t_{2} \rightarrow_{a}^{*} T_{2}
\end{aligned}
$$

Our goal now is to construct the reduction sequence:

$$
\Gamma_{\text {sub }}\left(t_{1} t_{2}\right) \rightarrow_{a}^{*}\left(T_{2} \Rightarrow T_{1}\right) \Gamma_{\text {sub }} t_{2} \rightarrow_{a}^{*}\left(T_{2} \Rightarrow T_{1}\right) T_{2} \rightarrow_{a} T_{1}
$$

To construct this sequence, it is sufficient to apply transitivity of $\rightarrow_{a}^{*}$ and Lemmas 4.5 and 4.6 .

Case:

$$
\frac{\Gamma, x: T \vdash t: T^{\prime}}{\Gamma \vdash \lambda x: T \cdot t: T \Rightarrow T^{\prime}}
$$

By the induction hypothesis on the premise of this rule, we have:

$$
\Gamma_{\text {sub }}[T / x] t \rightarrow_{a}^{*} T^{\prime}
$$

Now we need to show that

$$
\Gamma_{s u b}(\lambda x: T . t) \rightarrow_{a}^{*}\left(T \Rightarrow T^{\prime}\right)
$$

By applying one $a(\lambda)$ step and Lemma 4.7 we get:

$$
\Gamma_{\text {sub }}(\lambda x: T . t) \rightarrow_{a}\left(T \Rightarrow \Gamma_{\text {sub }}[T / x] t\right) \rightarrow_{a}^{*}\left(T \Rightarrow T^{\prime}\right)
$$

This requires the fact that $\Gamma_{\text {sub }}[T / x]=[T / x] \Gamma_{\text {sub }}$, which holds because $x \notin \operatorname{dom}\left(\Gamma_{\text {sub }}\right)$ since we may rename $x$ to avoid this, and because $T$ contains no term variables and hence is unaffected by applying $\Gamma_{\text {sub }}$. 
Proof of Theorem 4.4, right-to-left. Since abstract reduction is convergent (Theorems 4.2 and 4.3), we may assume that redexes in the reduction sequence to $T$ are always reduced in leftmost order. Note that convergence is sufficient to justify this assumption, as $T$ is a normal form, and hence any strategy is guaranteed to reduce the starting term to $T$ in a finite number of steps. This assumption will simplify some reasoning below. We assume $\left[T_{1} / x_{1}, \cdots, T_{n} / x_{n}\right] t \rightarrow_{a}^{*} T$ and prove $x_{1}: T_{1}, \cdots, x_{n}: T_{n} \vdash t: T$ by induction on the number $n$ of leftmost $\rightarrow_{a}$ steps in the reduction to $T$.

Base Case: there are no $\rightarrow_{a}$ steps. This means that our term $t$ cannot be reduced

$$
\Gamma_{\text {sub }} t=T
$$

In this case, $t$ must be a variable (or else substitution could not result in a type $T$ ). So, $t=x$ for some variable $x$, where $\Gamma(x)=T$. Then we get:

$$
\frac{\Gamma(x)=T}{\Gamma \vdash x: T}
$$

Step Case: there is at least one $\rightarrow_{a}$ step. We proceed by case splitting on the form of $t$.

Case:

$$
\Gamma_{\text {sub }} x
$$

This case cannot occur, since either $x \notin d o m\left(\Gamma_{\text {sub }}\right)$, in which case we cannot have $x \rightarrow_{a}^{*} T$ for any type $T$; or else $x \in \operatorname{dom}\left(\Gamma_{\text {sub }}\right)$, and then $\Gamma_{\text {sub }} x=T$. We cannot have a $\rightarrow_{a}$ step in that case, because types are normal forms for abstract reduction (Lemma 4.1).

Case:

$$
\Gamma_{s u b} f
$$

The only possible step is $f \rightarrow_{a} A \Rightarrow A$, and we indeed have $\Gamma \vdash f: A \Rightarrow A$. The case for $\Gamma_{\text {sub }} a$ is similar.

Case:

$$
\Gamma_{s u b}\left(t_{1} t_{2}\right)
$$

In this case, the reduction sequence must be of the following form, for some mixed term $t^{\prime}$ and type $T_{2}$, and some natural numbers $k_{1}$ and $k_{2}$ :

$$
\Gamma_{s u b}\left(t_{1} t_{2}\right) \rightarrow_{a}^{k_{1}}\left(\left(T_{2} \Rightarrow T\right) t_{2}\right) \rightarrow_{a}^{k_{2}}\left(T_{2} \Rightarrow T\right) T_{2} \rightarrow_{a} T
$$

where

$$
\begin{array}{llll}
\text { 1. } & \Gamma_{\text {sub }} t_{1} & \rightarrow_{a}^{k_{1}} & T_{2} \Rightarrow T \\
\text { 2. } & \Gamma_{\text {sub }} t_{2} & \rightarrow k_{a}^{k_{2}} & T_{2}
\end{array}
$$

We are justified in assuming this, because there must be some first position in the reduction sequence from $t_{1} t_{2}$ to $T$ where a descendant of $t_{1} t_{2}$ is reduced. That descendant here is $\left(T_{2} \Rightarrow T\right) T_{2}$. In the reduction sequence prior to that point, we are assuming (as noted at the start of the proof) that steps occur in leftmost order, so the $t_{1}$ steps come first, and then the $t_{2}$ ones. Now we can apply the induction hypothesis to (1) and (2), which each have shorter length than the original reduction sequence. This gives us the premises of the following inference, which suffices to complete this case:

$$
\frac{\Gamma \vdash t_{1}: T_{2} \Rightarrow T \quad \Gamma \vdash t_{2}: T_{2}}{\Gamma \vdash t_{1} t_{2}: T}
$$

\section{Case:}

$$
\Gamma_{s u b}\left(\lambda x: T^{\prime} \cdot t^{\prime}\right)
$$


In this case, we may assume the reduction sequence is of the following form, for some $T^{\prime \prime}$ :

$$
\Gamma_{\text {sub }}\left(\lambda x: T^{\prime} . t^{\prime}\right) \rightarrow_{a}\left(T^{\prime} \Rightarrow\left[T^{\prime} / x\right] \Gamma_{\text {sub }} t^{\prime}\right) \rightarrow_{a}^{*}\left(T^{\prime} \Rightarrow T^{\prime \prime}\right)
$$

where

$$
\left[T^{\prime} / x\right] \Gamma_{s u b} t^{\prime} \rightarrow_{a}^{*} T^{\prime \prime}
$$

This is because $\lambda x: T^{\prime} . t^{\prime}$ is itself an abstract redex, and since we are assuming our reduction is in leftmost, it must be reduced immediately. Now we can apply the induction hypothesis on $\left[T^{\prime} / x\right] \Gamma_{\text {sub }} t^{\prime} \rightarrow_{a}^{*} T^{\prime \prime}$ and get the premise of the following inference, which suffices to complete this case:

$$
\frac{\Gamma, x: T^{\prime} \vdash t^{\prime}: T^{\prime \prime}}{\Gamma \vdash \lambda x: T^{\prime} \cdot t^{\prime}: T^{\prime} \Rightarrow T^{\prime \prime}}
$$

\section{Generic Theorems for Preservation and Combined Confluence}

In this section, we collect some abstract properties for $\rightarrow_{a}$ and $\rightarrow_{b}$, from which type preservation and confluence of $\rightarrow_{a b}$ can be concluded. In subsequent sections we will instantiate these theorems with abstract and concrete reduction relations.

For the first theorem, recall that in our setting $\rightarrow_{a}$ computes the type of a term, or else could reach a stuck term like $(A \Rightarrow A)(A \Rightarrow A)$ which does not correspond to a type. We want to speak about reductions that lead to types, so we need to phrase the following theorem in terms of some set $S$, which we will instantiate later with a set of types. In condition (3) of the theorem, we interpose $I d_{\leftarrow a}^{*}(S)$ to restrict peaks to those objects which $a$-reduce to an object in $S$.

Theorem 5.1. Assume

(1) $\rightarrow_{a}(S)=\emptyset$ (that is, $S$ is a set of objects in normal form with respect to $\rightarrow_{a}$ ).

(2) $\rightarrow_{a}$ is confluent.

(3) $\leftarrow_{a} \cdot I d_{\leftarrow_{a}^{*}(S)} \cdot \rightarrow_{b} \subseteq\left(\rightarrow_{b} \cup \rightarrow_{a}^{*}\right) \cdot \leftarrow_{a}^{*}$; that is, for every $m$ such that there exists $T \in S$ with $m \rightarrow_{a}^{*} T$, and every $m^{\prime}$ and $m^{\prime \prime}$ with $m \rightarrow_{a} m^{\prime}$ and $m \rightarrow_{b} m^{\prime \prime}$, there exists a $m^{\prime \prime \prime}$ such that $m^{\prime \prime} \rightarrow_{a}^{*} m^{\prime \prime \prime}$ and either $m^{\prime} \rightarrow_{b} m^{\prime \prime \prime}$ or $m^{\prime} \rightarrow_{a}^{*} m^{\prime \prime \prime}$.

(4) every normal form with respect to $\rightarrow_{a}$ is also a normal form with respect to $\rightarrow_{b}$.

Then if $T \in S$ and $T \leftarrow_{a}^{*} m \rightarrow_{b} m^{\prime}$, we have $m^{\prime} \rightarrow_{a}^{*} T$.

Proof. Let $m \rightarrow_{a}^{*} T$ and $m \rightarrow_{b} m^{\prime}$, we have to prove that $m^{\prime} \rightarrow_{a}^{*} T$. We do this by induction on the number $n$ of steps in $m \rightarrow_{a}^{n} T$. In case $n=0$ we have $m=T$. By (1), $T$ is a normal form with respect to $\rightarrow_{a}$, which is a normal form with respect to $\rightarrow_{b}$ due to (4). So $m \rightarrow_{b} m^{\prime}$ is not possible, and the claim holds trivially.

For the induction step assume $m \rightarrow_{a} m_{1}$ for which $m_{1} \rightarrow_{a}^{n-1} T$. Applying (3) now yields $m_{3}$ such that $m^{\prime} \rightarrow_{a}^{*} m_{3}$ and either $m_{1} \rightarrow_{b} m_{3}$ or $m_{1} \rightarrow_{a}^{*} m_{3}$. In case $m_{1} \rightarrow_{b} m_{3}$ we apply the induction hypothesis on $m_{1} \rightarrow_{a}^{n-1} T$ and conclude $m^{\prime} \rightarrow_{a}^{*} m_{3} \rightarrow_{a}^{*} T$. In case $m_{1} \rightarrow_{a}^{*} m_{3}$ we apply confluence of $\rightarrow_{a}(2)$ by which $T$ and $m_{3}$ have a common $\rightarrow_{a}$-reduct. As $T$ is a normal form with respect to $\rightarrow_{a}$ by (1), we conclude $m^{\prime} \rightarrow_{a}^{*} m_{3} \rightarrow_{a}^{*} T$, concluding the proof. 
Lemma 5.2. Suppose $\rightarrow_{a}$ and $\rightarrow_{b}$ are binary relations such that

(1) $\rightarrow_{a}$ is confluent, and

$(2) \leftarrow_{a} \cdot \rightarrow_{b} \subseteq\left(\rightarrow_{b} \cup \rightarrow_{a}^{*}\right) \cdot \leftarrow_{a}^{*}$.

Then we also have

$$
\leftarrow_{a}^{*} \cdot \rightarrow_{b} \subseteq\left(\rightarrow_{b} \cup \rightarrow_{a}^{*}\right) \cdot \leftarrow_{a}^{*}
$$

Proof. Assume $t \rightarrow_{a}^{n} u$ and $t \rightarrow_{b} v$; we have to find $w$ such that $u \rightarrow_{b} \cup \rightarrow_{a}^{*} w$ and $v \rightarrow_{a}^{*} w$. We do this by induction on $n$. For $n=0$ we choose $w=v$. For $n>0$ write $t \rightarrow_{a} t^{\prime} \rightarrow_{a}^{n-1} u$. By (2) an element $v^{\prime}$ exists such that $v \rightarrow_{a}^{*} v^{\prime}$ and either $t^{\prime} \rightarrow_{a}^{*} v^{\prime}$ or $t^{\prime} \rightarrow_{b}$. If $t^{\prime} \rightarrow_{a}^{*} v^{\prime}$ we apply (1) yielding $w$ satisfying $u \rightarrow_{a}^{*} w$ and $v^{\prime} \rightarrow_{a}^{*} w$ and we are done. If $t^{\prime} \rightarrow_{b}$ then we apply the induction hypothesis yielding $u\left(\rightarrow_{b} \cup \rightarrow_{a}^{*}\right) w$ and $v^{\prime} \rightarrow_{a}^{*} w$.

Theorem 5.3. Let $\rightarrow_{a}$ and $\rightarrow_{b}$ be binary relations (recall from Section 2 that we write $\rightarrow_{b a}$ for $\rightarrow_{a} \cup \rightarrow_{b}$ ). Assume

(1) $\rightarrow_{a}$ is terminating,

(2) $\rightarrow_{a}$ is confluent,

(3) $\leftarrow_{a} \cdot \rightarrow_{b} \subseteq\left(\rightarrow_{b} \cup \rightarrow_{a}^{*}\right) \cdot \leftarrow_{a}^{*}$, and

(4) every normal form with respect to $\rightarrow_{a}$ is also a normal form with respect to $\rightarrow_{b}$.

Then $\rightarrow_{b a}$ is confluent.

Proof. By Lemma 5.2, we have:

$$
\left(3^{\prime}\right) \quad \leftarrow_{a}^{*} \cdot \rightarrow_{b} \subseteq\left(\rightarrow_{b} \cup \rightarrow_{a}^{*}\right) \cdot \leftarrow_{a}^{*} .
$$

Now let $t \rightarrow_{b a}^{*} u$ and $t \rightarrow_{b a}^{*} v$; for proving the theorem we have to prove that $w$ exists satisfying $u \rightarrow_{b a}^{*} w$ and $v \rightarrow_{b a}^{*} w$. Choose $w$ to be a $\rightarrow_{a}$-normal form of $t$, which exists due to (1). Assume $t \rightarrow_{b a}^{n} u$; we will prove that $u \rightarrow_{a}^{*} w$ by induction on $n$. For $n=0$ this follows from $t \rightarrow_{a}^{*} w$. For $n>0$ let $t \rightarrow_{b a}^{n-1} u^{\prime} \rightarrow_{b a} u$. From the induction hypothesis we conclude $u^{\prime} \rightarrow_{a}^{*} w$. Combining (2) and $\left(3^{\prime}\right)$ yields

$$
\leftarrow_{a}^{*} \cdot \rightarrow_{b a} \subseteq\left(\rightarrow_{b} \cup \rightarrow_{a}^{*}\right) \cdot \leftarrow_{a}^{*} .
$$

So since $w \leftarrow_{a}^{*} u^{\prime} \rightarrow_{b a} u$ we conclude that $w^{\prime}$ exists satisfying $w \rightarrow_{b} w^{\prime}$ or $w \rightarrow_{a}^{*} w^{\prime}$, and $u \rightarrow_{a}^{*} w^{\prime}$. Since $w$ is not only a $\rightarrow_{a}$-normal form, but also a $\rightarrow_{b}$-normal form according to (4), we conclude $w^{\prime}=w$. Hence $u \rightarrow_{a}^{*} w^{\prime}=w$, concluding the proof of $u \rightarrow_{a}^{*} w$. Applying the same argument on $t \rightarrow_{b a}^{*} v$ we conclude $v \rightarrow_{a}^{*} w$, concluding the proof of the theorem.

One may wonder whether the requirement of termination is essential for Theorem 5.3 . It is: on the set $\{1,2,3\}$ the relations $\rightarrow_{a}=\{(1,1)\}$ and $\rightarrow_{b}=\{(1,2),(1,3)\}$ satisfy all requirements of Theorem 5.3, while $\rightarrow_{b a}$ is not confluent.

One may wonder whether in Theorem 5.3 the condition (4) on normal forms is essential. It is, even if not only $\rightarrow_{a}$ is terminating and confluent but also $\rightarrow_{b}$, as is shown by the following example of relations on 10 elements, in which $\rightarrow_{a}$ steps are denoted by dashed arrows and $\rightarrow_{b}$ steps are denoted by solid arrows. 


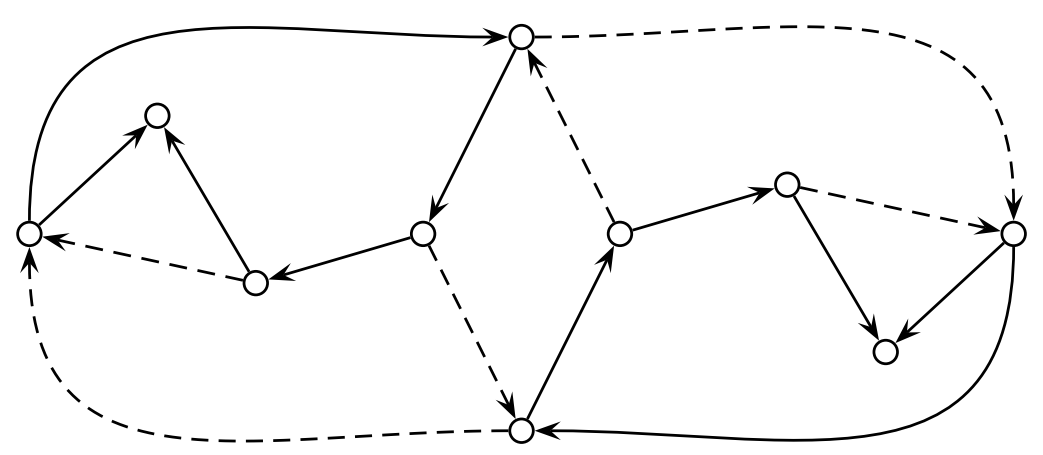

In this example there are two convertible normal forms, so the union is not confluent, and both $\rightarrow_{a}$ and $\rightarrow_{b}$ are both confluent and terminating; $\rightarrow_{a}$ is even deterministic. Also condition (3) of Theorem 5.3 is easily checked, even stronger: $\leftarrow_{a} \cdot \rightarrow_{b} \subseteq \rightarrow_{b a} \cdot \leftarrow_{a}^{=}$. This example was found using a SAT solver. A direct encoding of the example to be looked for run out of resources. However, by adding a symmetry requirement, was observed on the first example, the SAT solver yielded a satisfying assignment that could be interpreted as a valid example. The example given above was obtained from this after removing some redundant arrows. Independently, Bertram Felgenhauer found an example that could be simplified to exactly the same example as given here. This remarkable example was the starting point of developing the tool CARPA by which such examples can be found fully automatically.

\section{Type Preservation and Combined Confluence for STLC}

We now prove type preservation for full $\beta$-reduction (the $\rightarrow_{b}$ relation of Section 4), based on the rewriting formulation. This is in contrast to the results of Kuan et al., who obtain type preservation for the rewriting approach as a corollary of type preservation based on a standard big-step notion of typing (and the relation of that notion of typing with the small-step notion).

Definition 6.1 (Typability). A mixed term $m$ is called typable if $m \rightarrow_{a}^{*} T$ for some type $T$.

If we translate our standard statement of type preservation (at the beginning of Section 3.2) so that it uses abstract reduction instead of the usual typing relation, we have the following.

Theorem 6.2 (Type Preservation). Let $m, m^{\prime}$ be mixed terms and $T$ be a type. If $m \rightarrow_{a}^{*} T$ and $m \rightarrow_{b} m^{\prime}$, then $m^{\prime} \rightarrow_{a}^{*} T$.

The proof of this theorem is given by applying Theorem 5.1 we need to check its conditions (1), (2), (3) and (4). We instantiate the set $S$ in condition (1) with the set of types $T$, which are normal forms by Lemma 4.1. Condition (2) follows from Lemma 4.3. Condition (4) is immediate from the definitions of $\rightarrow_{a}$ and $\rightarrow_{b}$ : if $\rightarrow_{b}$ applies on a term $t$, then $t$ either contains $f a$ via rule $b(f-\beta)$ by which $\rightarrow_{a}$ applies via $a(f)$, or $t$ contains $\left.\lambda x: T . m\right]$ via rule $b \beta$ ) by which $\rightarrow_{a}$ applies via $a(\lambda)$. So it remains to check condition (3), which follows from the following lemma.

Lemma 6.3. Let $m_{0}$ be a typable mixed term and let $m_{1}, m_{2}$ be mixed terms such that $m_{0} \rightarrow_{a} m_{1}$ and $m_{0} \rightarrow_{b} m_{2}$. Then a mixed term $m_{3}$ exists such that $m_{2} \rightarrow_{a}^{*} m_{3}$ and either 
$m_{1} \rightarrow_{b} m_{3}$ or $m_{1} \rightarrow_{a}^{*} m_{3}$. Furthermore, if the step from $m_{0}$ to $m_{2}$ is a call-by-value step, so is the step from $m_{1}$ to $m_{3}$.

Proof. We distinguish the ways the redexes in $m_{0}$ are related.

If the redexes of $m_{0} \rightarrow_{a} m_{1}$ and $m_{0} \rightarrow_{b} m_{2}$ are parallel, then $m_{3}$ can be chosen such that $m_{1} \rightarrow_{b} m_{3}$ and $m_{2} \rightarrow_{a} m_{3}$ (preserving whether or not the $b$-step is call-by-value).

If the redex of $m_{0} \rightarrow_{a} m_{1}$ is above the redex of $m_{0} \rightarrow_{b} m_{2}$, then the $\rightarrow_{a}$ step is either of the type $a(\beta)$ or $a(\lambda)$, in which the $\rightarrow_{b}$ acts on the mixed term $m$ as it occurs in the rule $a(\beta)$ or $a(\lambda)$. As this $m$ is not duplicated, we get $m_{3}$ such that $m_{1} \rightarrow_{b} m_{3}$ and $m_{1} \rightarrow_{a} m_{3}$ (and the step $m_{0} \rightarrow_{b} m_{2}$ is not call-by-value).

If the redex of $m_{0} \rightarrow_{a} m_{1}$ is below the redex of $m_{0} \rightarrow_{b} m_{2}$, then some further case analysis is required.

If there is no overlap, then $m_{3}$ can be chosen such that $m_{1} \rightarrow_{b} m_{3}$ (preserving being call-by-value) and $m_{2} \rightarrow_{a}^{*} m_{3}$.

If there is overlap and $m_{0} \rightarrow_{a} m_{1}$ is an application of $a(f)$ or $a(a)$, then $m_{0}=E_{a}[f a]$ and $m_{2}=E_{a}[a]$, and $m_{3}$ can be chosen to be $E_{a}[A]$, satisfying $m_{1} \rightarrow_{a}^{2} m_{3}$ and $m_{2} \rightarrow_{a} m_{3}$.

The remaining case is illustrated by the following picture:

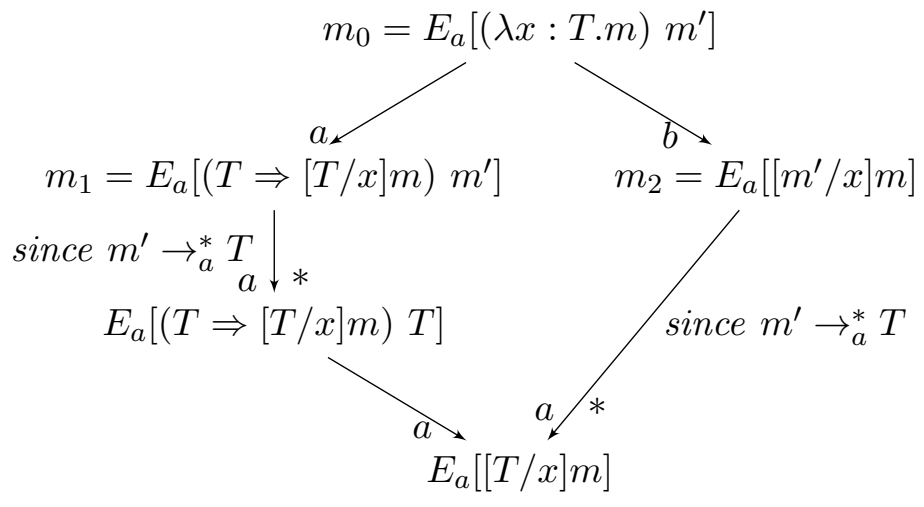

The picture already shows that by choosing $m_{3}=E_{a}[[T / x] m]$ we obtain $m_{1} \rightarrow_{a}^{*} m_{3}$ and $m_{2} \rightarrow_{a}^{*} m_{3}$ if we can prove $m^{\prime} \rightarrow_{a}^{*} T$. For doing so we use the assumption that $m_{0}$ is typable: there exists a type $T^{\prime}$ such that $m_{0}=E_{a}\left[(\lambda x: T . m) m^{\prime}\right] \rightarrow_{a}^{*} T^{\prime}$. Since $T^{\prime}$ is a type it does not contain a $\lambda$ symbol, so somewhere in this reduction the $\lambda$ in $\lambda x$ :T.m should be removed. By inspecting the rules we see that this can only be done by the rule $a(\lambda)$ by which $\lambda x: T$.- is replaced by $T \Rightarrow-$. Next the (invisible) application symbol in $\left(\lambda x:\right.$ T.m $m^{\prime}$ should be removed. This can only be done by the rule $a(\beta)$. This rule is only applicable if first $m^{\prime}$ is rewritten by $\rightarrow_{a}$ steps to $T$, indeed proving $m^{\prime} \rightarrow_{a}^{*} T$.

Theorem 6.4. The relation $\left(I d_{\leftarrow_{a}^{*}(\text { Types })} \cdot \rightarrow_{a}\right) \cup\left(I d_{\leftarrow_{a}^{*}(\text { Types })} \cdot \rightarrow_{b}\right)$ is confluent.

Proof. We will apply Theorem [5.3. For this, we need to check properties (1) to (4) for the particular relations $I d_{\leftarrow_{a}^{*} \text { (Types) }} \cdot \rightarrow_{a}$ and $I d_{\leftarrow_{a}^{*} \text { (Types) }} \cdot \rightarrow_{b}$. Property (2) follows from Theorem 4.3 and the fact that $I d_{\leftarrow a}^{*}$ (Types) is the identity relation. All peaks must be of the form $m_{1} \leftarrow_{a} m \leftarrow_{i d} m \rightarrow_{i d} m \rightarrow_{a} m_{2}$, due to the composition with $I d_{\leftarrow{ }_{a}^{*}(\text { Types })}$. By Theorem 4.3, if $m_{1} \leftarrow \leftarrow_{a} m \rightarrow_{a} m_{2}$, then there exists $m_{3}$ such that $m_{1} \rightarrow_{a}^{*} m_{3} \leftarrow a m_{2}$. Thus, any $I d_{\leftarrow * a}(\text { Types })^{\cdot} \rightarrow_{a}$ peak $m_{1} \leftarrow_{a} m \leftarrow{ }_{i d} m \rightarrow_{i d} m \rightarrow_{a} m_{2}$ can be completed with $m_{1} \rightarrow_{i d} m_{1} \rightarrow_{a} m_{3} \leftarrow_{a} m_{2} \leftarrow{ }_{i d} m_{2}$. Likewise, By Theorem $4.2 \rightarrow_{a}$ is terminating, so $I d_{\leftarrow{ }_{a}^{*}(\text { Types })} \cdot \rightarrow_{a} \subseteq \rightarrow_{a}$ is also terminating, proving property (1). Property (3) follows from 
Lemma 6.3. So it remains to prove Property (4). This is immediate from the definitions of $\rightarrow_{a}$ and $\rightarrow_{b}$ : if $\rightarrow_{b}$ applies on a term $t$, then $t$ either contains $f a$ via rule $b(f-\beta)$ by which $\rightarrow_{a}$ applies via $a(f)$, or $t$ contains $\lambda x: T . m$ via rule $b(\beta)$ by which $\rightarrow_{a}$ applies via $a(\lambda)$.

Corollary 6.5 (Confluence of Combined Reduction). Every typable mixed term is confluent with respect to the reduction relation $\rightarrow_{b a}$.

Proof. Confluence of the set of typable mixed terms is equivalent to confluence of the relation $I d_{\leftarrow \text { ( }(\text { Types })} \cdot \rightarrow_{b a}$, which is easily seen to be equal to

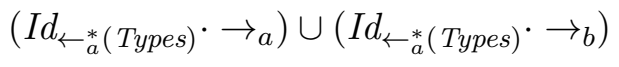

By Theorem 6.4, the latter relation is confluent.

A form of typability is essential, since the relation $\rightarrow_{b a}$ is not confluent in general, as Kuan et al. note also in their setting. For instance, the non-typable term $(\lambda x: A . x)(\lambda x$ : $A . x)$ has two distinct normal forms

$$
(A \Rightarrow A)(A \Rightarrow A) \leftarrow_{a}^{+}(\lambda x: A . x)(\lambda x: A . x) \rightarrow_{b} \lambda x: A . x \rightarrow_{a}(A \Rightarrow A) .
$$

\section{Progress and Type Safety for STLC}

In this section, we complete the basic meta-theory for STLC by proving progress and type safety theorems for call-by-value reduction (the $\rightarrow_{c}$ relation of Section 44). Lemmas 7.4 and 7.5 are stated in a somewhat more general way, so that we can also use them to show type safety for the generalized form of typability we will consider in Section 9 .

7.1. Quasi-Stuck Terms. We begin by inductively defining the set of quasi-stuck terms $S$, in Figure 5. Also, let us call a quasi-stuck term which is not a value stuck. The purpose of these definitions is to generalize a characterization of $c$-normal standard terms to mixed terms (Lemmas 7.1 and [7.2, proved next), in such a way that we can show that the set of quasi-stuck terms is closed under abstract reduction (Lemma 7.3, proved below). This will allow us to prove that typable quasi-stuck terms must be values (Lemma 7.5), from which we easily obtain the desired main theorems of progress and type safety.

Lemma 7.1. If $m$ is quasi-stuck, then $m t_{c}$.

Proof. The proof is by an easy structural induction on $m$, using the definition of quasistuck.

- Mixed values $u$ are in $S$.

- Terms of the form $a s$ or $A s$ are in $S$ if $s \in S$.

- Terms of the form $f s$ or $(A \Rightarrow A) s$ are in $S$ if $s \in S$ and $s$ is neither $a$ nor $A$.

- Terms of the form $(\lambda x: T . m) s$ or $(T \Rightarrow m) s$ are in $S$ if $s \in S$ and $s$ is not a mixed value.

- Terms of the form $s s^{\prime}$ are in $S$ if $s, s^{\prime} \in S$ and $s$ is not a mixed value.

Figure 5: Inductive definition of the set $S$ of quasi-stuck terms 
Lemma 7.2. If standard term $t$ is closed and $t \rightarrow_{c}$, then $t$ is quasi-stuck.

Proof. The proof is by structural induction on $t$. If $t$ is a (standard) value it is quasi-stuck, and it cannot be a variable since $t$ is closed. So suppose it is an application $t_{1} t_{2}$. Since $t_{1}$ is closed, $t_{1}$ cannot be a variable. We consider now the remaining possibilities. It could be that $t_{1}$ is $a$ and $t_{2}$ is some other $c$-normal form. Then by the induction hypothesis, $t_{2}$ is quasi-stuck, and $t$ is, too, using the second clause above in the definition of quasi-stuck terms. Next, we could have the situation where $t_{1}$ is $f$, and $t_{2}$ is any $c$-normal form except $a$. Then by the induction hypothesis, $t_{2}$ is quasi-stuck, and $t$ is, too, using the third clause in the definition of quasi-stuck terms. Next, we could have that $t_{1}$ is a $\lambda$-abstraction, and $t_{2}$ is any $c$-normal form except a standard value. Then by the induction hypothesis, $t_{2}$ is quasi-stuck, and it cannot be a mixed value other than a standard value, because $t_{2}$ is a standard term. So $t$ is quasi-stuck, too, using the fourth clause. Finally, if $t_{1}$ is some application, then by the induction hypothesis, $t_{1}$ and $t_{2}$ are both quasi-stuck. Since $t_{1}$ is not a value, the fifth clause above gives us that $t$ is quasi-stuck.

Lemma 7.3 (Reduction of Quasi-Stuck Terms). If $m$ is quasi-stuck, and $m \rightarrow_{a} m^{\prime}$, then $m^{\prime}$ is also quasi-stuck. Furthermore, if $m$ is a mixed value, then so is $m^{\prime}$; and if $m$ is not a mixed value, then neither is $m^{\prime}$.

Proof. The proof is by structural induction on $m$. Suppose $m$ is a mixed value. Then it is easy to see by inspection of the reduction rules that $m^{\prime}$ must be, too. So suppose $m$ is of the form $a s$ or $A s$ with $s \in S$. Then either the assumed reduction is of the form $a s \rightarrow_{a} A s$, or else of the form $a s \rightarrow_{a} a m^{\prime \prime}$ or $A s \rightarrow_{a} A m^{\prime \prime}$. In the former case, the resulting term is a quasi-stuck non-value. In the latter, we may apply the induction hypothesis to conclude that $m^{\prime \prime}$ is quasi-stuck, and hence $a m^{\prime \prime}$ (or $A m^{\prime \prime}$ ) is a quasi-stuck non-value.

If $m$ is of the form $f s$ or $(A \Rightarrow A) s$, where $s \in S$ and $s$ is not $a$ or $A$, then either the assumed reduction is of the form $f s \rightarrow_{a}(A \Rightarrow A) s$ or else $f s \rightarrow_{a} f m^{\prime \prime}$ or $(A \Rightarrow A) s \rightarrow_{a}$ $(A \Rightarrow A) m^{\prime \prime}$. In the former case, the resulting term is a quasi-stuck non-value, by the third clause of the definition of quasi-stuck terms above. In the latter, if $s$ is not a value, we again use our induction hypothesis to conclude that $m^{\prime \prime}$ is a quasi-stuck non-value, and hence not $a$ or $A$. So $m^{\prime}$ is a quasi-stuck non-value, too. If $s$ is a value, then so is $m^{\prime \prime}$, and reduction cannot turn a value other than $a$ into $a$ or $A$. So again, $m^{\prime \prime}$ has the required form to be a quasi-stuck non-value.

Suppose $m$ is of the form $\left(\lambda x: T \cdot m^{\prime \prime}\right) s$ or $\left(T \Rightarrow m^{\prime \prime}\right) s$, with $s \in S$ and $s$ not a mixed value. Then either the assumed reduction is of the form $\left(\lambda x: T \cdot m^{\prime \prime}\right) s \rightarrow_{a}\left(T \Rightarrow[T / x] m^{\prime \prime}\right) s$; or else of the form $\left(\lambda x: T \cdot m^{\prime \prime}\right) s \rightarrow_{a}\left(\lambda x: T \cdot m^{\prime \prime \prime}\right) s$ or $\left(T \Rightarrow m^{\prime \prime}\right) s \rightarrow_{a}\left(T \Rightarrow m^{\prime \prime \prime}\right) s$; or else of the form $\left(\lambda x: T . m^{\prime \prime}\right) s \rightarrow_{a}\left(\lambda x: T \cdot m^{\prime \prime}\right) m^{\prime \prime \prime}$ or $\left(T \Rightarrow m^{\prime \prime}\right) s \rightarrow_{a}\left(T \Rightarrow m^{\prime \prime}\right) m^{\prime \prime \prime}$. In the first two cases, the resulting term still has the required form to be a quasi-stuck non-value. In the third case, we know $s$ is not a value by the definition of quasi-stuck terms, so we may use our induction hypothesis to conclude that $m^{\prime \prime \prime}$ is a quasi-stuck non-value, which is sufficient to conclude that the resulting term is again stuck.

Finally, suppose $m$ is of the form $m_{1} m_{2}$, where $m_{1}$ is not a mixed value. Then the assumed reduction must be of the form either $m_{1} m_{2} \rightarrow_{a} m_{1}^{\prime} m_{2}$ or else $m_{1} m_{2} \rightarrow_{a} m_{1} m_{2}^{\prime}$, for some $m_{1}^{\prime}$ with $m_{1} \rightarrow_{a} m_{1}^{\prime}$, or else some $m_{2}^{\prime}$ with $m_{2} \rightarrow_{a} m_{2}^{\prime}$. This is because, by inspection of the reduction rules, $m$ itself cannot be a redex if $m_{1}$ is not a mixed value. In the former case, we may apply the induction hypothesis to conclude that $m_{1}^{\prime}$ is a quasistuck non-value, and hence so is $m^{\prime}$. In the latter, we may apply the induction hypothesis to conclude that $m_{2}^{\prime}$ is quasi-stuck, and hence so is $m^{\prime}$. 
Lemma 7.4. If $m$ is quasi-stuck (including the case where $m$ is a closed mixed value), and $m \rightarrow_{c a}^{*} T$, then $m \rightarrow_{a}^{*} T$.

Proof. The proof is by induction on the length of the reduction sequence from $m$ to $T$. If this length is 0 , the result obviously holds. So suppose we have $m \rightarrow_{c a} m^{\prime} \rightarrow_{c a}^{*} T$. Since $m$ is quasi-stuck, we have $m t_{c}$ by Lemma 7.1. So it must be the case that $m \rightarrow_{a} m^{\prime}$. Since $m^{\prime}$ is quasi-stuck by Lemma 7.3, we may apply our induction hypothesis to conclude $m^{\prime} \rightarrow_{a}^{*} T$, and hence $m \rightarrow_{a}^{*} T$.

Lemma 7.5. Suppose $m$ is a closed quasi-stuck term. Suppose further that $m \rightarrow_{c a}^{*} T$. Then $m$ is a mixed value.

Proof. The proof is similar to the previous one, and proceeds by induction on the length of the reduction sequence from $m$ to $T$. If this length is 0 , the result holds, since types are mixed values. So suppose we have $m \rightarrow_{c a} m^{\prime} \rightarrow_{c a}^{*} T$. Since $m$ is quasi-stuck, we have $m t_{c}$ by Lemma 7.1. So it must be the case that $m \rightarrow_{a} m^{\prime}$. We now consider cases on the form of $m$. If $m$ is a mixed value the result holds. So suppose it is a non-value. Then by Lemma 7.3 , $\mathrm{m}^{\prime}$ must also be a quasi-stuck non-value, and we may apply the induction hypothesis to derive a contradiction.

7.2. Concluding Progress and Type Safety. Armed with the concept of quasi-stuck terms and its associated lemmas, we can now obtain the main results of this section.

Theorem 7.6 (Progress). If standard term $t$ is closed, $t \rightarrow_{a}^{*} T$, and $t t_{c}$, then $t$ is a (standard) value.

Proof. By Lemma 7.2 and the assumption $t t_{c}$, we know $t$ is quasi-stuck. Now since our assumption that $t \rightarrow_{a}^{*} T$ implies $t \rightarrow_{c a}^{*} T$, we can apply Lemma 7.5 to conclude that $t$ is a mixed value (and hence a standard value, since $t$ is a standard term).

Theorem 7.7 (Type Safety). If standard term $t$ is closed, $t \rightarrow_{a}^{*} T$, and $t \rightarrow_{c}^{*} m t_{c}$, then $m$ is a standard value.

Proof. The proof is by induction on the length of the reduction sequence from $t$ to $m$. In the base case, we apply Theorems [7.6, since we have $m=t t_{c}$ in that case. For the step case, suppose we have $t \rightarrow_{c} m^{\prime} \rightarrow_{c}^{*} m t_{c}$. In this case, we can apply Theorem 6.2 to conclude $m^{\prime} \rightarrow_{a}^{*} T$. It is easily proved by induction on the structure of call-by-value evaluation contexts $E_{c}$ that if we have $t \rightarrow_{c} m^{\prime}$, then $m^{\prime}$ is a standard term $t^{\prime}$. We may now apply the induction hypothesis, since we have $t^{\prime} \rightarrow_{a}^{*} T$ and $t^{\prime} \rightarrow_{c} m t_{c}$.

\section{Applying Automated Analysis Tools to Type Preservation}

In this section, we show how automated tools for analyzing term-rewriting systems can be applied to automate part of the proof of type preservation. We will consider a language, which we call Uniform-STC, that does not distinguish terms and types syntactically. Advanced type systems like Pure Type Systems must often rely solely on the typing rules to distinguish terms and types (and kinds, superkinds, etc.) [5]. In Uniform-STC, we explore issues that arise in applying the rewriting approach to more advanced type systems. We must now implement kinding (i.e., type checking of types) as part of the abstract reduction 
mixed terms $t$ mixed values $u$
$::=S\left\langle t_{1}, t_{2}, t_{3}\right\rangle\left|K\left\langle t_{1}, t_{2}\right\rangle\right| t_{1} t_{2}\left|t_{1} \Rightarrow t_{2}\right| A \mid \operatorname{kind}\left(t_{1}, t_{2}\right)$ $::=S\left\langle t_{1}, t_{2}, t_{3}\right\rangle\left|K\left\langle t_{1}, t_{2}\right\rangle\right| A \mid t_{1} \Rightarrow t_{2}$

concrete evaluation contexts $E_{c}::=*\left|E_{c} t\right| u E_{c}$

Figure 6: Uniform-STLC language syntax and evaluation contexts

$$
\begin{array}{ll}
c(\beta-S) . & \overline{E_{c}\left[S\left\langle t_{1}, t_{2}, t_{3}\right\rangle u u^{\prime} u^{\prime \prime}\right] \rightarrow_{c} E_{c}\left[u u^{\prime \prime}\left(u^{\prime} u^{\prime \prime}\right)\right]} \\
c(\beta-K) . & \overline{E_{c}\left[K\left\langle t_{1}, t_{2}\right\rangle u u^{\prime}\right] \rightarrow_{c} E_{c}[u]} \\
a(S) . & S\left\langle t_{1}, t_{2}, t_{3}\right\rangle \rightarrow_{a} \operatorname{kind}\left(t_{1}, \operatorname{kind}\left(t_{2}, \operatorname{kind}\left(t_{3},\left(t_{1} \Rightarrow t_{2} \Rightarrow t_{3}\right) \Rightarrow\left(t_{1} \Rightarrow t_{2}\right) \Rightarrow\left(t_{1} \Rightarrow t_{3}\right)\right)\right)\right) \\
a(K) . & K\left\langle t_{1}, t_{2}\right\rangle \rightarrow_{a} \operatorname{kind}\left(t_{1}, \operatorname{kind}\left(t_{2},\left(t_{1} \Rightarrow t_{2} \Rightarrow t_{1}\right)\right)\right) \\
a(\beta) . & \left(t_{1} \Rightarrow t_{2}\right) t_{1} \rightarrow_{a} \operatorname{kind}\left(t_{1}, t_{2}\right) \\
a(k-\Rightarrow) . & \operatorname{kind}\left(\left(t_{1} \Rightarrow t_{2}\right), t\right) \rightarrow_{a} \operatorname{kind}\left(t_{1}, \operatorname{kind}\left(t_{2}, t\right)\right) \\
a(k-A) . \quad & \operatorname{kind}(A, t) \rightarrow_{a} t
\end{array}
$$

Figure 7: Concrete and abstract reduction rules

relation. We adopt a combinatory formulation so that the abstract reduction relation can be described by a first-order term-rewriting system.

Figure 6 shows the syntax for the Uniform-STC language. There is a single syntactic category $t$ for mixed terms and types, which include a base type $A$ and simple function types. $S\left\langle t_{1}, t_{2}, t_{3}\right\rangle$ and $K\left\langle t_{1}, t_{2}\right\rangle$ are the usual combinators, indexed by terms which determine their simple types. The kind construct for terms is used to implement kinding. The rules for concrete and abstract reduction are given in Figure 17. The concrete rules are just the standard ones for call-by-value reduction of combinator terms. For abstraction reduction, we are using first-order term-rewriting rules (unlike for previous systems).

For STLC (Section [6), abstract $\beta$-redexes have the form $(T \Rightarrow t) T$. For Uniform-STC, since there is no syntactic distinction between terms and types, abstract $\beta$-redexes take the form $\left(t_{1} \Rightarrow t_{2}\right) t_{1}$, and we must use kinding to ensure that $t_{1}$ is a type. This is why the $a(\beta)$ rule introduces a kind-term. We also enforce kinding when abstracting simply typed combinators $S\left\langle t_{1}, t_{2}, t_{3}\right\rangle$ and $K\left\langle t_{1}, t_{2}\right\rangle$ to their types. The rules for kind-terms $(a(k-\Rightarrow)$ and $a(k-A))$ make sure that the first term is a type, and then reduce to the second term.

Here, we define typability by value $u$ to mean abstract reduction to $u$ where $u$ is kindable, which we define as $\operatorname{kind}(u, A) \rightarrow_{a}^{*} A$. This definition avoids the need to define types syntactically.

Following the methodology embodied in Theorem 5.1, we must first prove the abstract reduction is confluent. In fact, it is convergent, and we can apply analysis tools to determine this, as shown in the next two theorems.

Theorem 8.1. The term rewriting system $\rightarrow_{a}$ is terminating.

Proof. The automated termination checker APROve reports that the rewrite system for $\rightarrow_{a}$ is terminating, using a recursive path ordering [11].

Theorem 8.2. The term rewriting system $\rightarrow_{a}$ is confluent. 
Proof. Abstract reduction for Uniform-STC does not have the diamond property due to the non-left-linear rule $a(\beta)$, where there could indeed be redexes in the expressions matching the repeated variable $t_{1}$. By Theorem 8.1, however, we can apply Newman's Lemma to conclude confluence from local confluence. Local confluence follows because all the $a a$-peaks can be joined using either one $a$-step on either side as for STLC, or else using additional balancing steps if one of the rules applied is $a(\beta)$.

But even easier than this reasoning is applying an automated confluence checker: the $\mathrm{ACP}$ tool immediately reports that the abstract reduction relation is confluent [3].

The proofs of Theorems 8.1 and 8.2 demonstrate how the rewriting approach to typing benefits from recent advances in analysis tools for term rewriting: we can use termination and confluence checkers to analyze the abstract reduction relation $\rightarrow_{a}$ corresponding to typing. We expect this situation to recur for more advanced type systems, although some may provide new challenges for automated analysis tools (we give an example below).

Lemma 8.3. $\leftarrow_{a} \cdot I d_{\leftarrow a}^{*}(S) \cdot \rightarrow_{c} \subseteq\left(\rightarrow_{c} \cup \rightarrow_{a}^{*}\right) \cdot \leftarrow_{a}^{*}$.

Proof. We distinguish the peaks originating at typable terms $t$.

If $\leftarrow_{a}$ and $\rightarrow_{c}$ steps are parallel $-E_{c}^{\prime}[t] \leftarrow_{a} E_{c}[t] \leftarrow_{i d} E_{c}[t] \rightarrow_{i d} E_{c}[t] \rightarrow_{c} E_{c}\left[t^{\prime}\right]-$ the peak can be completed directly $E_{c}^{\prime}[t] \rightarrow_{i d} E_{c}^{\prime}[t] \rightarrow_{c} E_{c}^{\prime}\left[t^{\prime}\right] \leftarrow{ }_{a} E_{c}\left[t^{\prime}\right] \leftarrow{ }_{i d} E_{c}\left[t^{\prime}\right]$.

If the $\leftarrow_{a}$ and $\rightarrow_{c}$ steps overlap, there are two cases, corresponding to $c(\beta-K)$ and $c(\beta-S)$ reduction steps. We show the completion for $c(\beta-K)$ peaks (omitting the $\rightarrow_{i d}$ steps to simplify the presentation); the argument for $c(\beta-S)$ peaks is similar.

$$
\begin{array}{ll}
P . & E_{c}\left[u\left[\left(\hat{t} t t^{\prime}\right)\right]\right] \leftarrow{ }_{a} E_{c}\left[\left(K\left\langle t_{1}, t_{2}\right\rangle t t^{\prime}\right)\right] \rightarrow \rightarrow_{a} E_{c}[t] \\
L . & E_{c}\left[u\left[\left(\hat{t} t t^{\prime}\right)\right]\right] \rightarrow \rightarrow_{a}^{*} E_{c}\left[u\left[\left(\hat{t} t_{1} t^{\prime \prime}\right)\right]\right] \rightarrow{ }_{a} E_{c}\left[u\left[\left(\left(t_{2} \Rightarrow t_{1}\right) t^{\prime \prime}\right)\right]\right] \rightarrow_{a}^{*} \\
& E_{c}\left[u\left[\left(\left(t_{2} \Rightarrow t_{1}\right) t_{2}\right)\right]\right] \rightarrow{ }_{a} E_{c}\left[\operatorname{kind}\left(t_{1}, \operatorname{kind}\left(t_{2}, t_{1}\right)\right)\right] \rightarrow_{a}^{*} E_{c}\left[t_{1}\right] \\
R . & E_{c}[t] \rightarrow{ }_{a}^{*} E_{c}\left[t_{1}\right]
\end{array}
$$

The $\rightarrow{ }_{a}^{*}$-steps are justified because the peak term (shown on line $(\mathrm{P})$ ) is typable by composition with $I d_{\leftarrow a}^{*}(S)$. By confluence of abstract reduction, this implies that the sources of all the left steps are also typable. For each $\rightarrow_{a}^{*}$-step, since abstract reduction cannot drop redexes (as all rules are non-erasing), we argue as for STLC that a descendant of the appropriate displayed kind-term or application must eventually be contracted, as otherwise, a stuck descendant of such would remain in the final term. Kindable terms cannot contain stuck applications or stuck kind-terms, because our abstract reduction rules are non-erasing. And contraction of those displayed kind-terms or applications requires the reductions used for the $\rightarrow_{a}^{*}$-steps, which are sufficient to complete the peak.

Lemma 8.4. Every normal form with respect to $\rightarrow_{a}$ is also a normal form with respect to $\rightarrow_{b}$.

The normal forms of $\rightarrow_{a}$ include $A, t_{1} \Rightarrow t_{2}$ where $t_{1}$ and $t_{2}$ are $a$-normal forms, $\left(t_{1} \Rightarrow t_{2}\right) t_{1}^{\prime}$ where $t_{1} \neq t_{1}^{\prime}$, and $\operatorname{kind}\left(t_{1}, t\right)$ where $t_{1}$ is not generated by the grammar $T::=A \mid T \Rightarrow T$. By inspection, $E_{c}[A] t_{c}$ and $E_{c}\left[t_{1} \Rightarrow t_{2}\right] t_{c}$.

Theorem 8.5 (Type Preservation). Let $m, m^{\prime}$ be mixed terms and $T$ be a term such that $k i n d(T, t) \rightarrow_{a}$ t. If $m \rightarrow_{a}^{*} T$ and $m \rightarrow_{c} m^{\prime}$, then $m^{\prime} \rightarrow_{a}^{*} T$.

Proof. By application of Theorem [5.1. Condition (1) is satisfied by instantiating $S$ by the set of terms $\left\{t \mid k i n d\left(t, t^{\prime}\right) \rightarrow_{a} t\right\}$. Condition (2) follows by Theorem 8.2. Condition (3) by Lemma 8.3, condition (4) by Lemma 8.4. 
Theorem 8.6. Every mixed typable term is confluent with respect to the reduction relation $\rightarrow a c$.

Proof. For proving that $\rightarrow_{b a}$ is confluent for typable mixed terms we need to check properties (1) to (4) of Theorem 5.3 for the particular relations $I d_{\leftarrow_{a}^{*}(S)} \cdot \rightarrow_{a}$ and $I d_{\leftarrow_{c}^{*}(T)^{*}} \rightarrow_{c}$. The composition of $\rightarrow_{a}$ and $\rightarrow_{b}$ with $I d_{\leftarrow{ }_{a}^{*}(S)}$ serves to ensure that we are only considering typable terms.

Property (2) follows from Theorem 8.2 and the fact that $I d_{\leftarrow a}^{*(T y p e s)}$ is the identity relation. All 1-step peaks of must be of the form $m \leftarrow m \rightarrow m$, due to the composition with $I d_{\leftarrow{ }_{a}^{*}(\text { Types })}$. By Theorem 8.2, if $m_{1} \leftarrow_{a} m \rightarrow_{a} m_{2}$, then there exists $m_{3}$ such that $m_{1} \rightarrow_{a}^{*} m_{3} \leftarrow{ }_{a} m_{2}$. Thus, any $I d_{\leftarrow \leftarrow^{*}(\text { Types })^{*}} \rightarrow_{a}$ peak $m_{1} \leftarrow \leftarrow_{a} m \leftarrow_{i d} m \rightarrow_{i d} m \rightarrow_{a} m_{2}$ can be completed with $m_{1} \rightarrow_{i d} m_{1} \rightarrow_{a} m_{3} \leftarrow_{a} m_{2} \leftarrow{ }_{i d} m_{2}$. By Theorem 8.1 $\rightarrow_{a}$ is terminating, so $I d_{\leftarrow{ }_{a}^{*}(\text { Types })^{*}} \rightarrow_{a} \subseteq \rightarrow_{a}$ is also terminating, proving property (1). Property (3) follows from Lemma 8.3. Property (4) follows from Lemma 8.4.

As an aside, note that a natural modification of this problem is out of the range of ACP, version 0.20. Suppose we are trying to group kind-checking terms so that we can avoid duplicate kind checks for the same term. For this, we may wish to permute kindterms, and pull them out of other term constructs. The following rules implement this idea, and can be neither proved confluent nor disproved by ACP, version 0.20. Just the first seven rules are also unsolvable by ACP.

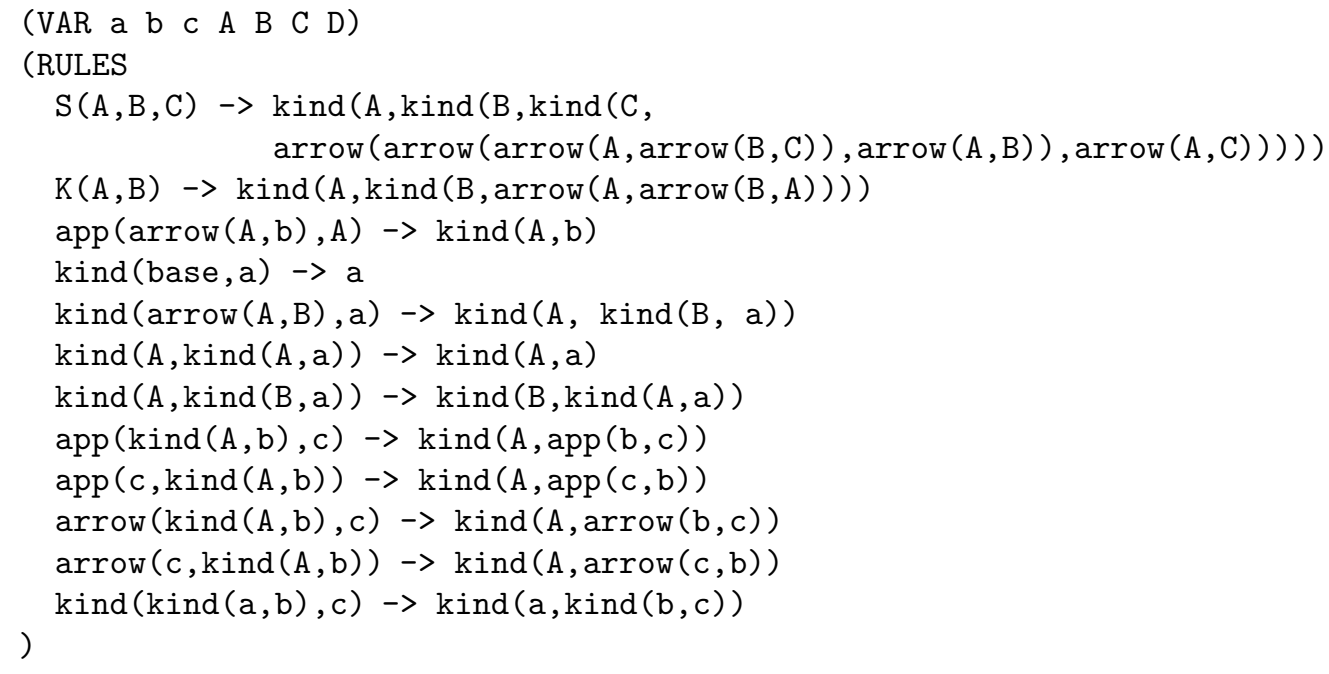

\section{Generalizing Nuprl's Direct Computation Rules}

Martin-Löf's Intuitionistic Type Theory (ITT), as formulated in [15], is a system of four judgments presented with a rigorous but informal semantics. A typing judgment of the form $a \in A$ "means that $a$ has a canonical object of the canonical type denoted by $\mathrm{A}$ as value" [15, page 174]. Here, Martin-Löf is making use of the concept of a term (of ITT) having a value, a concept he defines earlier in the paper. The authors of the Nuprl system realized that this semantics justifies more permissive typing rules than allowed by MartinLöf's own formal systems [8] (see also Section 2.2 of [2] for a historical perspective). In 
particular, it justifies so-called direct computation rules, which turned out to be useful for formal development with Nuprl:

$$
\frac{t \rightarrow^{*} t^{\prime} \quad t^{\prime} \in T}{t \in T}
$$

Applying Theorem 4.4, we can view this rule from a rewriting perspective. We will use callby-value reduction, as full $\beta$-reduction would require additional technicalities that would not be illuminating (we would have to use parallel reduction and incorporate a proof of confluence of $\beta$-reduction, in order to get preservation of generalized typing).

$$
\frac{t \rightarrow_{c}^{*} t^{\prime} t^{\prime} \rightarrow_{a}^{*} T}{t \rightarrow_{a}^{*} T}
$$

In this section, we will take the idea of Nuprl's direct computation rules one step further, by adopting the following definition.

Definition 9.1 (Generalized Typability). A mixed term $m$ is called generalized typable if $m \rightarrow_{c a}^{*} T$ for some type $T$.

This allows us to view (call-by-value versions of) Nuprl's direct computation rules as embodying a special case of generalized typability, namely $\rightarrow_{c}^{*} \cdot \rightarrow_{a}^{*}$. We will see in this section that we can prove type preservation directly for generalized typing, using the rewriting approach. Note that generalized typability is not obviously decidable, since $\rightarrow_{c a}$ is not terminating

A simple example of generalized typability is given by the term $(\lambda x: A . \lambda y: A . y) \lambda x$ : $A . x x$. Note that the argument term $\lambda x: A . x x$ is not simply typable. This term has several $c a$-reduction sequences, including the following one:

$$
\begin{aligned}
& (\lambda x: A . \lambda y: A . y) \lambda x: A . x x \rightarrow_{a} \\
& (\lambda x: A .(A \Rightarrow A)) \lambda x: A . x x \rightarrow_{a} \\
& (\lambda x: A .(A \Rightarrow A))(A \Rightarrow(A A)) \rightarrow_{c} \\
& A \Rightarrow A
\end{aligned}
$$

Because this term $c a$-reduces to a type, the generalized type-safety property we will obtain in this section tells us that the $c$-normal form of this term, if such exists, is a value. This can, of course, be confirmed for this case, where the $c$-normal form is just $\lambda y: A . y$. Notice that this example also shows that $\rightarrow_{c a}$ is not confluent, as we can also reduce it to a stuck term in this way:

$$
\begin{aligned}
& (\lambda x: A . \lambda y: A . y) \lambda x: A . x x \rightarrow_{a} \\
& (\lambda x: A .(A \Rightarrow A)) \lambda x: A \cdot x x \rightarrow_{a} \\
& (\lambda x: A .(A \Rightarrow A))(A \Rightarrow(A A)) \rightarrow_{a} \\
& (A \Rightarrow(A \Rightarrow A))(A \Rightarrow(A A)) \rightarrow_{c a}
\end{aligned}
$$

Theorem 9.2 (Generalized Type Preservation for Call-By-Value Reduction). If $m \rightarrow_{c a}^{*} T$ and $m \rightarrow_{c} m^{\prime}$, then $m^{\prime} \rightarrow_{c a}^{*} T$.

Proof. We cannot conveniently apply Theorem 5.1, because the natural instantiation would be to take $\rightarrow_{c a}$ for the relation $\rightarrow_{a}$ in the theorem - but then we would have to prove confluence of $\rightarrow_{c a}$, which does not hold (as shown just above). So instead we give a direct proof, by induction on the length of the assumed $a c$-sequence from $m$ to $T$. The sequence cannot be of length 0 , since $m$ cannot be a type (since it $c$-reduces, as no type can).

For the step case: suppose the assumed $c a$-reduction is of the form $m \rightarrow_{a} m^{\prime \prime} \rightarrow_{c a}^{*} T$. We now consider cases for the form of overlap of the step $m \rightarrow_{a} m^{\prime \prime}$ and $m \rightarrow_{c} m^{\prime}$. Suppose 
the $c$-step is $E_{c}[f a] \rightarrow_{c} E_{c}[a]$. If the $a$-step is in $E_{c}$, that means $m^{\prime \prime}=E_{c}^{\prime}[f a]$, where the hole in $E_{c}$ is at the same position as in $E_{c}^{\prime}$. We can just permute these steps, to obtain $E_{c}[a] \rightarrow_{a} E_{c}^{\prime}[a]$ and $E_{c}^{\prime}[f a] \rightarrow_{c} E_{c}^{\prime}[a]$. Now the induction hypothesis can be applied with $E_{c}^{\prime}\left[\begin{array}{ll}f & a\end{array}\right]$ (i.e., $m^{\prime \prime}$ ) as the peak term, and $E_{c}^{\prime}[a]$ as the term to which it $c$-steps.

So suppose the $a$-step is in the displayed $f a$ of $E_{c}[f a]$. Then before the reduction sequence from $m^{\prime \prime}$ to $T$ can perform a $c$-step, it must first reduce the residual of $f a$ to $A$, since that residual occurs in a $c$-reduction position. So the reduction sequence from $m^{\prime \prime}$ to $T$ must look like the following, where the hole in $E_{c}$ and in $E_{c}^{\prime}$ are at the same position:

$$
m^{\prime \prime} \rightarrow_{a}^{*} E_{c}^{\prime}[A] \rightarrow_{c a}^{*} T
$$

By performing the $a$-reductions which transformed $E_{c}$ to $E_{c}^{\prime}$, we can reduce $E_{c}[a]$ to $E_{c}^{\prime}[A]$, and then we are done, since we then have $m^{\prime} \rightarrow_{a}^{*} E_{c}^{\prime}[A] \rightarrow_{c a}^{*} T$.

We now must consider the case where the $c$-step is $E_{c}\left[\left(\lambda x: T^{\prime} \cdot m_{1}\right) u\right] \rightarrow_{c} E_{c}\left[[u / x] m_{1}\right]$. Again, if the $a$-step is in $E_{c}$, we can permute steps and apply the induction hypothesis. If the $a$-step is in $m_{1}$ or in $u$, we can also permute the steps, though if the reduction is in $u$ (say $u \rightarrow_{a} u^{\prime}$ ), we will in general have $E_{c}\left[[u / x] m_{1}\right] \rightarrow_{a}^{*} E_{c}\left[\left[u^{\prime} / x\right] m_{1}\right]$, since $x$ need not appear exactly once in $m_{1}$. Nevertheless, we can still apply the induction hypothesis with $m^{\prime \prime}$ as the peak term, since we will only ever produce one $c$-step from $m^{\prime \prime}$ by permuting steps. Finally, suppose the $a$-step is $E_{c}\left[\left(\lambda x: T^{\prime} \cdot m_{1}\right) u\right] \rightarrow_{a} E_{c}\left[\left(T^{\prime} \Rightarrow\left[T^{\prime} / x\right] m_{1}\right) u\right]$. By similar reasoning as in the previous case, the $c a$-reduction sequence from $E_{c}\left[\left(T^{\prime} \Rightarrow\left[T^{\prime} / x\right] m_{1}\right) u\right]$ to $T$ may contain $a$-steps transforming $E_{c}$ to some $E_{c}^{\prime}$, but it cannot take a $c$-step until it has reduced the displayed $\left(T^{\prime} \Rightarrow\left[T^{\prime} / x\right] m_{1}\right) u$ to $\left[T^{\prime} / x\right] m_{1}^{\prime}$, with $u \rightarrow_{a}^{*} T^{\prime}$ and $m_{1} \rightarrow_{a}^{*} m_{1}^{\prime}$. This is because that displayed term is in $c$-reduction position and neither a value nor a redex. We can then duplicate any $a$-steps taken in $E_{c}$ to $a$-reduce $E_{c}\left[[u / x] m_{1}\right]$ (i.e., $\left.m^{\prime}\right)$ to $E_{c}^{\prime}\left[\left[T^{\prime} / x\right] m_{1}^{\prime}\right]$. This term then $a c$-reduces to $T$, and we are done.

Theorem 9.3 (Generalized Progress). If standard term $t$ is closed, $t \rightarrow_{c a}^{*} T$, and $t t_{c}$, then $t$ is a (standard) value.

Proof. As for Theorem 7.6, we obtain this result by applying Lemmas 7.2 and 7.5 ,

Theorem 9.4 (Generalized Type Safety). If standard term $t$ is closed, $t \rightarrow_{c a}^{*} T$, and $t \rightarrow_{c}^{*} t^{\prime} t_{c}$, then $t^{\prime}$ is a (standard) value.

Proof. This is a direct corollary of Theorems 9.2 and 9.3 .

\section{A Rewriting Approach to Normalization for StLC}

In this Section, we will see how the rewriting approach to typing impacts a standard approach to proving that every typable (closed) standard term of the simply typed lambda calculus has a $b$-normal form. We will work with a slightly different presentation of STLC than we saw in Section 4, in particular dispensing with the term constants $a$ and $f$. We assume a non-empty set of type constants $A$. The syntax we are using in this section is:

$$
\begin{array}{ll}
\text { types } T & :=A \mid T_{1} \Rightarrow T_{2} \\
\text { mixed terms } m & ::=x|\lambda x: T \cdot m| m m^{\prime}|A| T \Rightarrow m \\
\text { standard terms } t & ::=x|\lambda x: T . t| t t^{\prime}
\end{array}
$$


The abstract and concrete reduction relations are then defined as follows, where we use mixed terms $m$ as contexts (sometimes using meta-variable $\hat{m}$ in this case), writing $m\left[m^{\prime}\right]$ to denote the replacement of the unique occurrence of a special variable $*$ in $m$ by $m^{\prime}$.

$$
\begin{aligned}
& \frac{\hat{m}\left[(\lambda x: T . m) m^{\prime}\right] \rightarrow_{b} \hat{m}\left[\left[m^{\prime} / x\right] m\right]}{} b(\beta) \\
& \frac{\hat{m}[(T \Rightarrow m) T] \rightarrow_{a} \hat{m}[m]}{a} a(\beta) \\
& \overline{\hat{m}[\lambda x: T . m] \rightarrow_{a} \hat{m}[T \Rightarrow[T / x] m]} a(\lambda)
\end{aligned}
$$

10.1. Interpretation of Mixed Terms. The proof in this section is based on ideas from standard proofs, such as Girard's proof in the book Proofs and Types [12]. The technical details evolve differently, however, since we are using the rewriting approach to typing. Similarly to Girard's proof, we are going to define an interpretation of open types as sets of standard terms. Here, we need to generalize this to give interpretations $\llbracket m \rrbracket_{\phi}$ of mixed terms $m$, where (as standard) $\phi$ assigns interpretations to the free variables of $m$. The most enlightening observation that will come from this is Theorem 10.6 (Abstraction Theorem), which says that interpretation is monotonic with respect to abstract reduction: if $m \rightarrow_{a} m^{\prime}$, then $\llbracket m \rrbracket_{\phi} \subseteq \llbracket m^{\prime} \rrbracket_{\phi}$. If one views a set as abstracting its elements, and if one considers a mixed term as a code for the set of terms which is its interpretation, then the Abstraction Theorem shows that more abstract codes have more abstract interpretations. This is an elegant perspective that arises - from the standard Tait-Girard method - only by taking a small-step view of typing; existing proofs for normalization in the literature do not have any theorem which corresponds (in any obvious way) to the Abstraction Theorem.

So now to begin the development, let WN be the set of standard terms which are weakly normalizing with respect to $\rightarrow_{b}$ (that is, terms $t$ such that there exists some $t^{\prime}$ such that $\left.t \rightarrow_{b}^{*} t^{\prime} f_{b}\right)$. Also, if $\rightarrow$ is any binary relation on standard terms and $R$ any set of standard terms, we will write $\rightarrow(R)$ for the image of $R$ under $\rightarrow$ (that is, $\left\{t^{\prime} \mid \exists t \in R . t \rightarrow t^{\prime}\right\}$ ).

We first define $\mathcal{R}$ to be the set of all sets $R$ of standard terms satisfying the following conditions:

$(1) \leftarrow_{b}^{*}(R) \subseteq R$

(2) $R \neq \emptyset$

(3) $R \subseteq W N$

The first condition ensures that $t^{\prime} \rightarrow_{b}^{*} t$ and $t \in R$ imply $t^{\prime} \in R$. An assumption like this is often made about such sets of terms. We will call elements of $\mathcal{R}$ reducibility sets. Much work has been devoted to comparing different conditions for families of sets in the context of the interpretation of types (see, e.g., [18, 10]). Our focus here is not so much on the specific conditions on the interpretations of mixed terms, as on how interpretations of terms in the abstract reduction relation are related. The conditions we adopt here are simple and sufficient for weak normalization of closed terms (cf. also Chapter 12 of [17]).

We will use $\phi$ as a meta-variable for assignments, which are functions from $\operatorname{Var}$ to $\mathcal{R}$. We write $\phi[R / x]$ to mean the function $\phi$ updated to map variable $x$ to $R \in \mathcal{R}$. Now for any $m$ and $\phi$ with $F V(m) \subseteq \operatorname{dom}(\phi)$, we define the interpretation $\llbracket m \rrbracket_{\phi}$ of $m$ with respect to $\phi$ in Figure 8. To ensure that interpretations of types satisfy the first property above 


$$
\begin{array}{ll}
\llbracket T \Rightarrow m \rrbracket_{\phi} & =\left\{t \mid \forall t^{\prime} \in \llbracket T \rrbracket_{\phi} \cdot t t^{\prime} \in \llbracket m \rrbracket_{\phi}\right\} \\
\llbracket x \rrbracket_{\phi} & =\phi(x) \\
\llbracket A \rrbracket_{\phi} & =\mathrm{WN} \\
\llbracket \lambda x: T . m \rrbracket_{\phi} & =\leftarrow_{b}^{*}\left(\left\{\lambda x: T . t \mid \forall t^{\prime} \in \llbracket T \rrbracket_{\phi} \cdot\left[t^{\prime} / x\right] t \in \llbracket m \rrbracket_{\left.\phi \llbracket \llbracket T \rrbracket_{\phi} / x\right]}\right\}\right) \\
\llbracket m_{1} m_{2} \rrbracket_{\phi} & =\leftarrow{ }_{b}^{*}\left(\left\{t_{1} t_{2} \mid t_{1} \in \llbracket m_{1} \rrbracket_{\phi} \wedge t_{2} \in \llbracket m_{2} \rrbracket_{\phi}\right\}\right.
\end{array}
$$

Figure 8: The interpretation of mixed terms

of reducibility sets, we need to close under $\leftarrow_{b}^{*}$ in the last two clauses of the definition (in Figure 8). Since we are proving normalization, we take the set of normalizing terms as the interpretation of $A$, similarly to what is standardly done for atomic types (e.g., in Girard's proof).

10.2. Interpretations of Types are Reducibility Sets. In this section, we prove that for all types $T$ and $\phi$ with $F V(T) \subseteq \operatorname{dom}(\phi)$, we have $\llbracket T \rrbracket_{\phi} \in \mathcal{R}$. We will elide this condition relating $T$ (or instead $m$ ) and $\phi$ below. We prove the three properties of reducibility sets given in the previous section. The properties must be proved in order, as later properties depend on earlier ones. The first property is needed in a more general form, for any mixed term $m$, and not just types $T$. The second two properties are only needed for types. The proofs in this section are similar to those used for the standard definition of typing, except that there, they are usually proved by mutual induction. Here we can prove them independently, though in sequence, due to the simpler form of the second property. While the development in this section is similar to the usual one, in the next section we will see something significantly different.

Lemma 10.1. $\leftarrow_{b}^{*} \llbracket m \rrbracket_{\phi} \subseteq \llbracket m \rrbracket_{\phi}$

Proof. The proof is by structural induction on $m$. If $m$ is a $\lambda$-abstraction, or application, the desired property follows by idempotence of $\leftarrow_{b}^{*}$ as an operator on sets of terms. If $m$ is a variable $x$, then the property follows by the same property for $\phi(x)$, since we stipulated assignments map variables to elements of $\mathcal{R}$. If $\phi=A$, then we must prove

$$
\leftarrow_{b}^{*}(W N) \subseteq \mathrm{WN}
$$

But this just amounts to the obvious fact that if $t^{\prime} \rightarrow_{b}^{*} t$ and $t$ is weakly normalizing, then $t^{\prime}$ is also weakly normalizing.

Finally, suppose $m$ is $T \Rightarrow m^{\prime}$ for some $m^{\prime}$. Assume an arbitrary $t \in \llbracket T \Rightarrow m^{\prime} \rrbracket_{\phi}$, and arbitrary $t^{\prime}$ with $t^{\prime} \rightarrow_{b}^{*} t$. We must show $t^{\prime} \in \llbracket T \Rightarrow m^{\prime} \rrbracket_{\phi}$. To do this, by the definition of the interpretation of $\Rightarrow$-terms, it suffices to consider arbitrary $t^{\prime \prime} \in \llbracket T \rrbracket_{\phi}$, and show $t^{\prime} t^{\prime \prime} \in \llbracket m^{\prime} \rrbracket_{\phi}$. We have $t t^{\prime \prime} \in \llbracket m^{\prime} \rrbracket_{\phi}$ by the definition of the interpretation of $\Rightarrow$-terms. Then we get the desired conclusion by the induction hypothesis on $m^{\prime}$, since $t t^{\prime \prime} \rightarrow_{b}^{*} t^{\prime} t^{\prime \prime}$.

Lemma 10.2. $\llbracket T \rrbracket_{\phi} \neq \emptyset$

Proof. The proof is by structural induction on $T$. If $T$ is $A$, then the desired property holds immediately, since $x$ is in WN $=\llbracket A \rrbracket_{\phi}$. So suppose $T \equiv T_{1} \Rightarrow T_{2}$, for some $T_{1}$ and $T_{2}$. We must exhibit some $t \in \llbracket T_{1} \Rightarrow T_{2} \rrbracket_{\phi}$. By the induction hypothesis applied to $T_{2}$, there exists some $t^{\prime} \in \llbracket T_{2} \rrbracket_{\phi}$. Now take $\lambda x: T_{1} . t^{\prime}$ for the required term $t$, where we assume $x \notin F V\left(t^{\prime}\right)$. We just have to confirm that $\lambda x: T_{1} \cdot t^{\prime} \in \llbracket T_{1} \Rightarrow T_{2} \rrbracket_{\phi}$. So assume arbitrary $t^{\prime \prime} \in \llbracket T_{1} \rrbracket_{\phi}$, 
and show $\left(\lambda x: T_{1} \cdot t^{\prime}\right) t^{\prime \prime} \in \llbracket T_{2} \rrbracket_{\phi}$. By Lemma 10.1, it suffices to prove $t^{\prime} \in \llbracket T_{2} \rrbracket_{\phi}$, since $\left(\lambda x: T_{1} \cdot t^{\prime}\right) t^{\prime \prime} \rightarrow_{b}^{*} t^{\prime}$. But we are assuming $t^{\prime} \in \llbracket T_{2} \rrbracket_{\phi}$.

Lemma 10.3. $\llbracket T \rrbracket_{\phi} \subseteq \mathrm{WN}$

Proof. The proof is again by structural induction on $T$, and is trivial when $T$ is $A$. So suppose $T \equiv T_{1} \Rightarrow T_{2}$, and assume arbitrary $t \in \llbracket T_{1} \Rightarrow T_{2} \rrbracket_{\phi}$. We must show $t \in \mathrm{WN}$. By Lemma 10.2, we know there exists some term $t^{\prime} \in \llbracket T_{1} \rrbracket_{\phi}$. Then by the definition of the interpretation of $\Rightarrow$-terms, $t t^{\prime} \in \llbracket T_{2} \rrbracket_{\phi}$. By the induction hypothesis applied to $T_{2}$, we then have $t t^{\prime} \in \mathrm{WN}$. But this implies $t \in \mathrm{WN}$, as required.

Corollary 10.4. $\llbracket T \rrbracket_{\phi} \in \mathcal{R}$

The above lemmas have proved that $\llbracket T \rrbracket_{\phi}$ satisfies the three properties for membership in $\mathcal{R}$. In the next section, we will also need the following lemma, whose proof is routine and omitted:

Lemma 10.5 (Semantic Substitution). $\llbracket[T / x] m \rrbracket_{\phi}=\llbracket m \rrbracket_{\phi\left[\llbracket T \rrbracket_{\phi} / x\right]}$

10.3. The Abstraction Theorem. In this section, we prove a remarkable theorem, from which the normalization property for typable terms will follow as a corollary. For any mixed terms $m$ and $m^{\prime}$, and any $\phi$ with $F V(m) \subseteq \operatorname{dom}(\phi)$, we have:

Theorem 10.6 (Abstraction Theorem). $m \rightarrow_{a} m^{\prime} \Longrightarrow \llbracket m \rrbracket_{\phi} \subseteq \llbracket m^{\prime} \rrbracket_{\phi}$

Note that well-definedness of $\llbracket m^{\prime} \rrbracket_{\phi}$ in the statement of the theorem follows from the assumption about $\phi$ and the observation that abstract reduction cannot introduce new variables.

This theorem is remarkable because it reflects the essence of abstraction: the gathering of different concrete entities under the same abstract one. The Abstraction Theorem shows that abstract reduction is increasing the set of concrete terms which are collected under a mixed (and so partially abstract) term. In the next section, we will see how to conclude normalization from this theorem.

Proof of Theorem 10.6. It suffices to prove by structural induction on $\hat{m}$ that for all $\phi$ and for all $m$ and $m^{\prime}$ where $m$ is a redex and $m^{\prime}$ its contractum:

$$
\hat{m}[m] \rightarrow_{a} \hat{m}\left[m^{\prime}\right] \longrightarrow \llbracket \hat{m}[m] \rrbracket_{\phi} \subseteq \llbracket \hat{m}\left[m^{\prime}\right] \rrbracket_{\phi}
$$

Case: $\hat{m} \equiv m_{1} m_{2}$, where the hole is in $m_{1}$. The case where the hole is in $m_{2}$ is similar, so we omit it. To show the required $\llbracket m_{1}[m] m_{2} \rrbracket_{\phi} \subseteq \llbracket m_{1}\left[m^{\prime}\right] m_{2} \rrbracket_{\phi}$, consider arbitrary $t \in \llbracket m_{1}[m] m_{2} \rrbracket_{\phi}$. By the definition of the interpretation of applications, we must have $t_{1} \in \llbracket m_{1}[m] \rrbracket_{\phi}$ and $t_{2} \in \llbracket m_{2} \rrbracket_{\phi}$ with $t \rightarrow_{b}^{*} t_{1} t_{2}$. Now by the induction hypothesis applied to $m_{1}$ we have:

$$
\llbracket m_{1}[m] \rrbracket_{\phi} \subseteq \llbracket m_{1}\left[m^{\prime}\right] \rrbracket_{\phi}
$$

This implies $t_{1} t_{2} \in \llbracket m_{1}\left[m^{\prime}\right] m_{2} \rrbracket_{\phi}$. From this, we obtain the desired $t \in \llbracket m_{1}\left[m^{\prime}\right] m_{2} \rrbracket_{\phi}$ by the definition of the interpretation of applications.

Case: $\hat{m} \equiv \lambda x: T . m_{1}$, for some $x, T$, and $m_{1}$, with the hole in $m_{1}$. Consider an arbitrary $t \in \llbracket \lambda x: T \cdot m_{1}[m] \rrbracket_{\phi}$. By the definition of the interpretation of $\lambda$-abstractions, this implies that there exists a term $t_{1}$ such that $t \rightarrow_{b}^{*} \lambda x: T . t_{1}$ and for all $t^{\prime \prime} \in \llbracket T \rrbracket_{\phi}$, we have $\left[t^{\prime \prime} / x\right] t_{1} \in$ 
$\llbracket m_{1}[m] \rrbracket_{\phi\left[\llbracket T \rrbracket_{\phi} / x\right]}$. We must show $t \in \llbracket \lambda x: T \cdot m_{1}\left[m^{\prime}\right] \rrbracket_{\phi}$. By the definition of the interpretation of $\lambda$-terms and Lemma 10.1, it suffices to prove $\left(\lambda x: T . t_{1}\right) t^{\prime \prime} \in \llbracket m_{1}\left[m^{\prime}\right] \rrbracket_{\phi\left[\llbracket T \rrbracket_{\phi} / x\right]}$ for arbitrary $t^{\prime \prime} \in \llbracket T \rrbracket_{\phi}$. Again applying Lemma 10.1, we can see it suffices to prove $\left[t^{\prime \prime} / x\right] t_{1} \in$ $\llbracket m_{1}\left[m^{\prime}\right] \rrbracket_{\phi\left[\llbracket T \rrbracket_{\phi} / x\right]}$. This now follows by the induction hypothesis applied to context $m_{1}$.

Case: $\hat{m}=*$. Now we must distinguish the two cases for an abstract reduction.

Case 1. Suppose that we have

$$
\lambda x: T . m \rightarrow_{a} T \Rightarrow[T / x] m
$$

We must prove $\llbracket \lambda x: T . m \rrbracket_{\phi} \subseteq \llbracket T \Rightarrow[T / x] m \rrbracket_{\phi}$. So assume arbitrary $t \in \llbracket \lambda x: T . m \rrbracket_{\phi}$, and show $t \in \llbracket T \Rightarrow[T / x] m \rrbracket_{\phi}$. To show that, it suffices to consider arbitrary $t^{\prime \prime} \in \llbracket T \rrbracket_{\phi}$, and prove $t t^{\prime \prime} \in \llbracket[T / x] m \rrbracket_{\phi}$. By the definition of the interpretation of $\lambda$-abstractions, we have $t \rightarrow_{b}^{*} \lambda x: T . t^{\prime}$, for some $t^{\prime}$, with $\left[t^{\prime \prime} / x\right] t^{\prime} \in \llbracket m \rrbracket_{\phi\left[\llbracket T \rrbracket_{\phi} / x\right]}$ for all $t^{\prime \prime} \in \llbracket T \rrbracket_{\phi}$. Since $t t^{\prime \prime} \rightarrow_{b}^{*}\left[t^{\prime \prime} / x\right] t^{\prime}$, it suffices by Lemma 10.1 just to prove $\left[t^{\prime \prime} / x\right] t^{\prime} \in \llbracket[T / x] m \rrbracket_{\phi}$. This follows from the fact just derived, applying also Lemma 10.5 .

Case 2. Suppose that we have

$$
(T \Rightarrow m) T \rightarrow_{a} m
$$

Assume an arbitrary $t \in \llbracket(T \Rightarrow m) T \rrbracket_{\phi}$. By the definition of the interpretation of applications, we then have that there exists $t_{1} \in \llbracket T \Rightarrow m \rrbracket_{\phi}$ and $t_{2} \in \llbracket T \rrbracket_{\phi}$ such that $t \rightarrow_{b}^{*} t_{1} t_{2}$. We must show $t \in \llbracket m \rrbracket_{\phi}$. By the definition of the interpretation of $\Rightarrow$-terms, we obtain $t_{1} t_{2} \in \llbracket m \rrbracket_{\phi}$. By Lemma 10.1, this suffices to establish $t \in \llbracket m \rrbracket_{\phi}$, since $t \rightarrow_{b}^{*} t_{1} t_{2}$.

10.4. Concluding Normalization. Using the Abstraction Theorem, we can obtain the main result that typable terms are normalizing. First, we need this helper lemma stating that standard terms are in their own interpretations:

Lemma 10.7. Consider an arbitrary standard term $t$ and assignment $\phi$, as well as function $\sigma$ from variables to standard terms. Suppose also that for all $x \in F V(t)$, we have $\sigma(x) \in$ $\phi(x)$. Then we have $\sigma t \in \llbracket t \rrbracket_{\phi}$.

Proof. The proof is by structural induction on $t$. If $t$ is a variable $x$, then we have $\sigma x \in \phi(x)$ by assumption. If $t$ is of the form $\lambda x: T . t_{1}$, then the definition of the interpretation of mixed terms tells us:

$$
\llbracket \lambda x: T . t_{1} \rrbracket_{\phi}=\leftarrow_{b}^{*}\left(\left\{\lambda x: T \cdot t^{\prime} \mid \forall t^{\prime \prime} \in \llbracket T \rrbracket_{\phi} \cdot\left[t^{\prime \prime} / x\right] t^{\prime} \in \llbracket t_{1} \rrbracket_{\phi}\right\}\right)
$$

To show that $\sigma \lambda x: T . t_{1}$ is itself a member of the set on the right-hand side of this equation, it suffices to consider an arbitrary $t^{\prime \prime} \in \llbracket T \rrbracket_{\phi}$, and show $\left[t^{\prime \prime} / x\right]\left(\sigma t_{1}\right) \in \llbracket t_{1} \rrbracket_{\phi\left[\llbracket T \rrbracket_{\phi} / x\right]}$. Here we can apply the induction hypothesis for $t_{1}$, with $\sigma\left[t^{\prime \prime} / x\right]$ and $\phi\left[\llbracket T \rrbracket_{\phi} / x\right]$. The two substitutions still satisfy the required properties. Finally, if $t$ is of the form $t_{1} t_{2}$, the result easily follows from the induction hypothesis applied to $t_{1}$ and also to $t_{2}$, and the definition of the interpretation of applications. 
Theorem 10.8 (Normalization for Typable Terms). For all closed standard terms $t$ and types $T$, if $t \rightarrow_{a}^{*} T$, then $t \in \mathrm{WN}$.

Proof. By Lemma 10.7, we have $t \in \llbracket t \rrbracket_{\emptyset}$. Then by iterated application of Theorem 10.6, we know that $\llbracket t \rrbracket_{\emptyset} \subseteq \llbracket T \rrbracket_{\emptyset}$. By Lemma 10.3, $\llbracket T \rrbracket_{\emptyset} \subseteq \mathrm{WN}$. Putting these facts together, we get this chain of relationships, which suffices:

$$
t \in \llbracket t \rrbracket_{\emptyset} \subseteq \llbracket T \rrbracket_{\emptyset} \subseteq \mathrm{WN}
$$

10.5. Summary of The Standard Proof. Here, we summarize Girard's proof of strong normalization, for purposes of comparison [12. This proof is based on the usual judgment $\Gamma \vdash t: T$ for STLC. One first defines an interpretation of types:

$$
\begin{array}{ll}
t \in \operatorname{Red}_{b} & \Leftrightarrow t \in S N \\
t \in \operatorname{Red}_{T \rightarrow T^{\prime}} & \Leftrightarrow \quad \forall t^{\prime} \in \operatorname{Red}_{T} .\left(t t^{\prime}\right) \in \operatorname{Red}_{T^{\prime}}
\end{array}
$$

This does not require use of a function $\phi$ as above (though the standard proof for System $\mathrm{F}$ does). For this interpretation of types, one then proves these three properties, by mutual structural induction on the type $T$ mentioned in all three properties:

(1) $\operatorname{Red}_{T}(t) \Rightarrow S N(t)$.

(2) $\operatorname{Red}_{T}(t) \Rightarrow \operatorname{Red}_{T}(n \operatorname{next}(t))$.

(3) If $t$ is neutral, then $\operatorname{Red}_{T}(n \operatorname{ext}(t)) \Rightarrow \operatorname{Red}_{T}(t)$.

A term is neutral iff it is not a $\lambda$-abstraction. The third property implies that all the variables are in $\operatorname{Red}_{T}$ for every $T$. Finally, one derives the following different theorem in place of the Abstraction Theorem:

Theorem 10.9 (Reducibility). Suppose $\left\{x_{1}: T_{1}, \ldots, x_{n}: T_{n}\right\} \vdash t: T$, and consider arbitrary $t_{i} \in \operatorname{Red}_{T_{i}}$, for all $i \in\{1, \ldots, n\}$. Then $\left[t_{1} / x_{1}, \ldots, t_{n} / x_{n}\right] t \in \operatorname{Red}_{T}$.

Now we can obtain as a corollary that $\Gamma \vdash t: T$ implies $t \in S N$, since $\operatorname{Red}_{b} \subseteq S N$ by the first property above, and a substitution $\sigma$ replacing $x$ by $x$ satisfies the required condition, since all variables are included in all sets $\operatorname{Red}_{T}$.

10.6. Discussion. The main difference in the rewriting-based development and the standard one is in deriving the Abstraction Theorem. The form of the theorem is completely different from Theorem 10.9. One nice technical feature is that for the proof of the Abstraction Theorem, we did not need to apply a substitution to terms inhabiting interpretations of types, as we did for Theorem 10.9. We still needed to use the idea of such a substitution, but it appeared only in a simple helper lemma, namely Lemma 10.7. This is an advantage of the rewriting-based version, since the substitution does not clutter up the proof of the central result. One disadvantage of the rewriting-based version is that we needed the function $\phi$ and Lemma 10.5- but this is not such a significant disadvantage, since those devices are needed when we move to System F in the standard development anyway. 


\section{Conclusion}

We have seen how rewriting techniques can be used to develop the meta-theory of simple types. Typing is treated as a small-step abstract reduction relation, and type safety, based on type preservation and progress theorems, can be established by analysis of the interactions between abstract and concrete reduction steps. A crucial ingredient of our approach to type preservation, as defined by Theorem [5.1, is to have a confluent abstract reduction relation. For simply typed lambda calculus, this was a trivial matter, but we saw a more complex example, where applying automated confluence-checking tools developed in the term-rewriting community was able to automate this part of the type preservation proof. Confluence of the combination of abstract and concrete reduction for typable terms is an easy corollary of type preservation (Theorem 5.3). We have also seen how to adapt a standard proof of normalization for simply typed terms, for the rewriting approach to typing. For this proof, mixed terms are interpreted as sets of standard terms, and the crucial insight is embodied in the Abstraction Theorem, which shows that those sets are enlarged by reduction of the corresponding mixed terms.

There are many avenues for future work. First, the rewriting approach should be applied to more advanced type systems, including ones with impredicative polymorphism. Dependent type systems pose a particular challenge, because from the point of view of abstract reduction, $\Pi$-bound variables must play a dual role. When computing a dependent function type $\Pi x: T . T^{\prime}$ from an abstraction $\lambda x: T . t$, we may need to abstract $x$ to $T$, as for STLC; but we may also need to leave it unabstracted, since with dependent types, $x$ is allowed to appear in the range type $T^{\prime}$. It would also be interesting to see if there are consequences of the rewriting approach to typing when applied to proofs via the Curry-Howard isomorphism. Theorem 10.6 (Abstraction) shows how the set of proofs in the meaning of a mixed proof term (part proof and part formula) increases as the term is abstracted. Certainly, the present methods yield the syntactic capability to incrementally transform a proof to the theorem it proves. This could already be valuable in practice for efficient proof checking, for example of large proofs produced by SAT or SMT solvers (cf. [21]).

It would be interesting to go further in automating proofs of type preservation based on the rewriting approach. While the Programming Languages community has invested substantial effort in recent years on computer-checked proofs of properties like type safety for programming languages (initiated particularly by the POPLmark Challenge [4]), there is relatively little work on fully automatic proofs of type preservation (an example is [19]). The rewriting approach could contribute to filling that gap, since the methods we used above for analyzing interactions of abstract and concrete steps to prove type preservation are similar to those used for proving confluence of combined reduction.

Our longer term goal is to use this approach to design and analyze type systems for symbolic simulation. In program verification tools like PEX and KEY, symbolic simulation is a central component [6, 23]. But these systems do not seek to prove that their symbolicsimulation algorithms are correct. Indeed, the authors of the KEY system argue against expending the effort to do this [7]. The rewriting approach promises to make it easier to relate symbolic simulation, viewed as an abstract reduction relation, with the small-step operational semantics.

Acknowledgments. We thank the anonymous LMCS reviewers for their very detailed comments, and a number of technical suggestions which have greatly improved this paper; 
and also participants of the RTA 2011 conference for their helpful feedback and suggestions about this work.

\section{REFERENCES}

[1] S. Abramsky, D. Gabbay, and T. Maibaum, editors. Handbook of Logic in Computer Science. Oxford University Press, 1992.

[2] Stuart F. Allen, Mark Bickford, Robert L. Constable, Richard Eaton, Christoph Kreitz, Lori Lorigo, and E. Moran. Innovations in computational type theory using Nuprl. J. Applied Logic, 4(4):428-469, 2006.

[3] T. Aoto, J. Yoshida, and Y. Toyama. Proving Confluence of Term Rewriting Systems Automatically. In R. Treinen, editor, Rewriting Techniques and Applications (RTA), pages 93-102, 2009.

[4] B. Aydemir, A. Bohannon, M. Fairbairn, J. Foster, B. Pierce, P. Sewell, D. Vytiniotis, G. Washburn, S. Weirich, and S. Zdancewic. Mechanized metatheory for the masses: The POPLmark Challenge. In Proceedings of the Eighteenth International Conference on Theorem Proving in Higher Order Logics (TPHOLs 2005), 2005.

[5] H. Barendregt. Lambda Calculi with Types. In S. Abramsky, D. Gabbay, and T. Maibaum, editors, Handbook of Logic in Computer Science, volume 2, pages 117-309. Oxford University Press, 1992.

[6] B. Beckert, R. Hähnle, and P. Schmitt, editors. Verification of Object-Oriented Software: The KeY Approach. LNCS 4334. Springer-Verlag, 2007.

[7] B. Beckert and V. Klebanov. Must Program Verification Systems and Calculi be Verified? In Proceedings, 3rd International Verification Workshop (VERIFY), Workshop at Federated Logic Conferences (FLoC), Seattle, USA, pages 34-41, 2006.

[8] Robert L. Constable, Stuart F. Allen, S. F. Allen, H. M. Bromley, W. R. Cleaveland, J. F. Cremer, R. W. Harper, Douglas J. Howe, T. B. Knoblock, N. P. Mendler, P. Panangaden, Scott F. Smith, James T. Sasaki, and S. F. Smith. Implementing Mathematics with The Nuprl Proof Development System. Prentice Hall, 1986.

[9] C. Ellison, T. Şerbănuţă, and G. Roşu. A Rewriting Logic Approach to Type Inference. In A. Corradini and U. Montanari, editors, Recent Trends in Algebraic Development Techniques (WADT), pages 135151, 2008.

[10] Jean Gallier. On Girard's "Candidats De Reductibilité", pages 123-230. Academic Press, 1990.

[11] J. Giesl, P. Schneider-Kamp, and R. Thiemann. Automatic Termination Proofs in the Dependency Pair Framework. In U. Furbach and N. Shankar, editors, Automated Reasoning, Third International Joint Conference (IJCAR), pages 281-286, 2006.

[12] J.-Y. Girard, Y. Lafont, and P. Taylor. Proofs and Types. Cambridge University Press, 1989.

[13] M. Hills and G. Rosu. A Rewriting Logic Semantics Approach to Modular Program Analysis. In C. Lynch, editor, Proceedings of the 21st International Conference on Rewriting Techniques and Applications, RTA 2010, July 11-13, 2010, Edinburgh, Scotland, UK, pages 151-160, 2010.

[14] G. Kuan, D. MacQueen, and R. Findler. A rewriting semantics for type inference. In Proceedings of the 16th European conference on Programming (ESOP), pages 426-440. Springer-Verlag, 2007.

[15] P. Martin-Löf and Z. A. Lozinski. Constructive Mathematics and Computer Programming. Philosophical Transactions of the Royal Society of London. Series A, Mathematical and Physical Sciences, 312(1522):pp. 501-518, 1984.

[16] J. Mitchell. Foundations for Programming Languages. The MIT Press, 1996.

[17] B. Pierce. Types and Programming Languages. The MIT Press, 2002.

[18] Colin Riba. Strong Normalization as Safe Interaction. In 22nd IEEE Symposium on Logic in Computer Science (LICS 2007), 10-12 July 2007, Wroclaw, Poland, Proceedings, pages 13-22. IEEE Computer Society, 2007.

[19] C. Schürmann and F. Pfenning. Automated Theorem Proving in a Simple Meta-Logic for LF. In C. Kirchner and H. Kirchner, editors, 15th International Conference on Automated Deduction (CADE), pages 286-300, 1998.

[20] Aaron Stump, Garrin Kimmell, and Roba El Haj Omar. Type Preservation as a Confluence Problem. In Manfred Schmidt-Schauß, editor, Proceedings of the 22nd International Conference on Rewriting Techniques and Applications (RTA), volume 10 of LIPIcs, pages 345-360, 2011. 
[21] Aaron Stump, Duckki Oe, Andrew Reynolds, Liana Hadarean, and Cesare Tinelli. Smt proof checking using a logical framework. Formal Methods in System Design, pages 1-28. available online as of July, 2012.

[22] TeReSe, editor. Term Rewriting Systems, volume 55 of Cambridge Tracts in Theoretical Computer Science. Cambridge University Press, 2003.

[23] N. Tillmann and W. Schulte. Parameterized Unit Tests. SIGSOFT Softw. Eng. Notes, 30:253-262, 2005.

[24] A. Wright and M. Felleisen. A Syntactic Approach to Type Soundness. Information and Computation, 115(1):38-94, 1994. 